This item was submitted to Loughborough's Research Repository by the author.

Items in Figshare are protected by copyright, with all rights reserved, unless otherwise indicated.

Differential-geometric approach to the integrability of hydrodynamic chains: the Haantjes tensor

PLEASE CITE THE PUBLISHED VERSION

LICENCE

CC BY-NC-ND 4.0

REPOSITORY RECORD

Ferapontov, E.V., and D.G. Marshall. 2019. "Differential-geometric Approach to the Integrability of Hydrodynamic Chains: The Haantjes Tensor”. figshare. https://hdl.handle.net/2134/387. 


\title{
Differential-geometric approach to the integrability of hydrodynamic chains: the Haantjes tensor
}

\author{
E.V. Ferapontov and D.G. Marshall \\ Department of Mathematical Sciences \\ Loughborough University \\ Loughborough, Leicestershire LE11 3TU \\ United Kingdom \\ e-mails: \\ E.V.Ferapontov@lboro.ac.uk \\ D.G.Marshall@lboro.ac.uk
}

\begin{abstract}
The integrability of $m$-component systems of hydrodynamic type, $\mathbf{u}_{t}=V(\mathbf{u}) \mathbf{u}_{x}$, by the generalized hodograph method requires the diagonalizability of the $m \times m$ matrix $V(\mathbf{u})$. This condition is known to be equivalent to the vanishing of the corresponding Haantjes tensor. We generalize this approach to hydrodynamic chains - infinite-component systems of hydrodynamic type for which the $\infty \times \infty$ matrix $V(\mathbf{u})$ is 'sufficiently sparse'. For such systems the Haantjes tensor is well-defined, and the calculation of its components involves finite summations only. We illustrate our approach by classifying broad classes of conservative and Hamiltonian hydrodynamic chains with the zero Haantjes tensor. We prove that the vanishing of the Haantjes tensor is the necessary condition for a hydrodynamic chain to possess an infinity of semi-Hamiltonian hydrodynamic reductions, thus providing an easyto-verify necessary condition for the integrability.
\end{abstract}

MSC: 35L40, 35L65, 37K10.

Keywords: Hydrodynamic chains, Haantjes tensor, Hydrodynamic Reductions. 


\section{Introduction}

Hydrodynamic chains are quasilinear first order PDEs of the form

$$
\mathbf{u}_{t}=V(\mathbf{u}) \mathbf{u}_{x}
$$

where $\mathbf{u}=\left(u^{1}, u^{2}, \ldots\right)^{t}$ is an infinite-component column vector and $V(\mathbf{u})$ is an $\infty \times \infty$ matrix. The classical example is the Benney chain (or Benney's moment equations),

$$
u_{t}^{n}=u_{x}^{n+1}+(n-1) u^{n-1} u_{x}^{1},
$$

$n=1,2, \ldots$, which was first derived in [2] from the equations for long nonlinear waves on a free surface. It was observed later in $[43,15]$ that the same system (2) results from a kinetic Vlasov equation. The system (2) has been thoroughly investigated in the subsequent publications $[32,23,24,27,43,44,40,15]$ where, in particular, its Hamiltonian and integrability aspects were uncovered. Hydrodynamic reductions of the chain (2) were studied in [16, 17, 18, 30]. Various deformations of Benney's equations are known. These include the modified Benney chain,

$$
u_{t}^{n}=u_{x}^{n+1}+u^{1} u_{x}^{n}+(n-1) u^{n} u_{x}^{1},
$$

obtained in [26] as a quasiclassical limit of the modified KP hierarchy. Its two-parameter deformation,

$$
u_{t}^{n}=u_{x}^{n+1}+u^{1} u_{x}^{n}+(a(n-1)+b) u^{n} u_{x}^{1},
$$

was constructed in [25] along with further examples of Hamiltonian integrable chains possessing complete systems of commuting integrals. Another deformation scheme, based on the $R$-matrix approach, was proposed in [3], see also [28, 29]. The specialization of the chain (4) corresponding to $a=0, b=-1$,

$$
u_{t}^{n}=u_{x}^{n+1}+u^{1} u_{x}^{n}-u^{n} u_{x}^{1}
$$

naturally appears in the theory of finite-gap solutions of integrable hierarchies of the KdV type $[1,31]$. Reductions of the chain (5), both hydrodynamic and differential, were extensively investigated in $[33,38,39]$. The case $a=1, b=2$ arises from the kinetic model for rarefied bubbly flows [20], see also [41] for an alternative representation of this chain.

A broad class of new examples was found in [34], see also [4], based on the symmetry approach. These papers provide a complete classification of integrable conservative chains of the form

$$
u_{t}^{1}=u_{x}^{2}, \quad u_{t}^{2}=g\left(u^{1}, u^{2}, u^{3}\right)_{x}, \quad u_{t}^{3}=h\left(u^{1}, u^{2}, u^{3}, u^{4}\right)_{x}, \ldots,
$$

etc. Requiring that the chain (6) is embedded into a commutative hierarchy of special type, it was proved in [34] that the function $g\left(u^{1}, u^{2}, u^{3}\right)$ uniquely determines all other equations of the chain (6), as well as the whole associated hierarchy. Moreover, the function $g\left(u^{1}, u^{2}, u^{3}\right)$ was shown to satisfy an over-determined involutive system of third order PDEs whose generic solution was expressed in terms of theta functions and the Chazy equation.

Our approach to the integrability of hydrodynamic chains is motivated by the theory of finite-component systems of hydrodynamic type, that is, equations of the form (1) where $\mathbf{u}=$ $\left(u^{1}, u^{2}, \ldots, u^{m}\right)^{t}$ is a $m$-component column vector and $V(\mathbf{u})=v_{j}^{i}(\mathbf{u})$ is an $m \times m$ matrix. Explicitly, one has

$$
u_{t}^{i}=v_{j}^{i}(\mathbf{u}) u_{x}^{j}, \quad i, j=1, \ldots, m
$$


Such systems naturally occur in applications in gas dynamics, fluid mechanics, chemical kinetics, Whitham averaging procedure, differential geometry and topological field theory. We refer to $[42,6,7]$ for a further discussion and references. It is known that many particularly important systems of the form (7) are diagonalizable, that is, reducible to the Riemann invariant form

$$
R_{t}^{i}=\lambda^{i}(\mathbf{R}) R_{x}^{i}
$$

where the characteristic speeds $v^{i}(\mathbf{R})$ satisfy the so-called semi-Hamiltonian property [42],

$$
\partial_{k}\left(\frac{\partial_{j} \lambda^{i}}{\lambda^{j}-\lambda^{i}}\right)=\partial_{j}\left(\frac{\partial_{k} \lambda^{i}}{\lambda^{k}-\lambda^{i}}\right)
$$

$\partial_{k}=\partial / \partial R^{k}, i \neq j \neq k$. We emphasize that the semi-Hamiltonian property (9) is usually automatically satisfied for diagonalizable systems of the 'physical' origin. For instance, a conservative diagonalizable system is necessarily semi-Hamiltonian, e.g. [37]. Such systems possess infinitely many conservation laws and commuting flows of hydrodynamic type and can be linearized by the generalized hodograph method [42]. Their analytic, differential-geometric and Hamiltonian aspects are well-understood by now.

Remarkably, there exists the effective tensor criterion of the diagonalizability which does not require the actual computation of eigenvalues and eigenvectors of the matrix $v_{j}^{i}$. Let us first calculate the Nijenhuis tensor of the matrix $v_{j}^{i}$,

$$
N_{j k}^{i}=v_{j}^{p} \partial_{u^{p}} v_{k}^{i}-v_{k}^{p} \partial_{u^{p}} v_{j}^{i}-v_{p}^{i}\left(\partial_{u^{j}} v_{k}^{p}-\partial_{u^{k}} v_{j}^{p}\right),
$$

(the standard summation convention over repeated indices is adopted), and introduce the Haantjes tensor

$$
H_{j k}^{i}=N_{p r}^{i} v_{j}^{p} v_{k}^{r}-N_{j r}^{p} v_{p}^{i} v_{k}^{r}-N_{r k}^{p} v_{p}^{i} v_{j}^{r}+N_{j k}^{p} v_{r}^{i} v_{p}^{r} .
$$

For strictly hyperbolic systems the condition of diagonalizability is given by the following theorem.

Theorem 1 [19] A hydrodynamic type system with mutually distinct characteristic speeds is diagonalizable if and only if the corresponding Haantjes tensor (11) is identically zero.

Since components of the Haantjes tensor can be calculated (using computer algebra) in any coordinate system, this provides the effective diagonalizability criterion.

Our main observation is that both tensors (10) and (11) make perfect sense for infinite matrices which are 'sufficiently sparse'. To be more rigorous, let us give the following

Definition 1. An infinite matrix $V(\mathbf{u})$ is said to belong to the class $C$ (chain class) if it satisfies the following two natural properties:

(a) each row of $V(\mathbf{u})$ contains finitely many nonzero elements;

(b) each matrix element of $V(\mathbf{u})$ depends on finitely many variables $u^{i}$.

Notice that the chains (2) - (6) clearly belong to the class $C$. For matrices from the class $C$ all contractions in the expressions (10) and (11) reduce to finite summations so that each particular component, say $H_{j k}^{i}$, is a well-defined object which can be effectively computed. Moreover, for a fixed value of the upper index $i$ there exist only finitely many non-zero components $H_{j k}^{i}$.

We propose the following 
Definition 2. A hydrodynamic chain from the class $C$ is said to be diagonalizable if all components of the corresponding Haantjes tensor (11) are zero.

We point out that the chains (2) - (5) are diagonalizable in this sense. Notice that our approach is entirely 'intrinsic': it does not require the knowledge of any 'extrinsic' objects such as commuting flows, Hamiltonian structures, Lax pairs, etc.

The vanishing of the Haantjes tensor turns out to be the effective classification criterion. As an elementary example let us consider the chain

$$
u_{t}^{n}=u_{x}^{n+1}+u^{1} u_{x}^{n}+c_{n} u^{n} u_{x}^{1}
$$

where $c_{n}=$ const. One can verify that the vanishing of the Haantjes tensor implies the recurrence relation $c_{n+2}=2 c_{n+1}-c_{n}$. Setting $c_{1}=b, c_{2}=a+b$ we recover the integrable chain (4).

Based on the same criterion, in Sect. 2 we classify diagonalizable chains of the type (6). It turns out that the conditions $H_{j k}^{1}=0$ are already sufficiently restrictive and imply an overdetermined system expressing all second order partial derivatives of the function $h$ is terms of $g$, see (13). The conditions of consistency of these equations lead to a closed-form involutive system expressing all third order partial derivatives of $g$ in terms of its lower order derivatives, see (14). We emphasize that exactly the same system was derived in [34] using the symmetry approach, as well as in [13] based on the method of hydrodynamic reductions. Thus, for conservative chains (6) the condition of diagonalizability is equivalent to the integrability. The requirement of the vanishing of other components $H_{j k}^{i}, i \geq 2$, impose no additional constraints on $h$ and $g$ : these conditions reconstruct the remaining equations of the chain (6). For instance, the conditions $H_{j k}^{2}=0$ specify the right hand side of the fourth equation $u_{t}^{4}=\ldots$, etc.

The characterization of diagonalizable chains of a more general form,

$$
u_{t}^{1}=f\left(u^{1}, u^{2}\right)_{x}, \quad u_{t}^{2}=g\left(u^{1}, u^{2}, u^{3}\right)_{x}, \quad u_{t}^{3}=h\left(u^{1}, u^{2}, u^{3}, u^{4}\right)_{x}, \ldots,
$$

etc, is proposed in Sect. 3. As in the previous example, the conditions $H_{j k}^{1}=0$ imply the expressions for all second order partial derivatives of $h$ in terms of $g$ and $f$. The conditions of consistency of these equations result in an involutive system expressing all third order partial derivatives of $g$ and $f$ in terms of lower order derivatives.

The classification of diagonalizable Hamiltonian chains of the form

$$
\mathbf{u}_{t}=\left(B \frac{d}{d x}+\frac{d}{d x} B^{t}\right) \frac{\partial h}{\partial \mathbf{u}}
$$

here $B^{i j}=(i-1) u_{i+j-2}$ and $h\left(u^{1}, u^{2}, u^{3}\right)$ is the Hamiltonian density, is given in Sect. 4. The Benney chain (2) corresponds to $h=\left(u^{3}+\left(u^{1}\right)^{2}\right) / 2$, see [24]. We have found a broad family of new Hamiltonian densities, in particular, $h=\left(u^{3}+P\left(u^{1}, u^{2}\right)\right)^{1 / 3}$ where $P$ is a certain cubic polynomial, thus extending the results of [25].

In Sect. 5 we prove that the condition of diagonalizability is necessary for the existence of 'sufficiently many' hydrodynamic reductions. Recall that an $m$-component hydrodynamic reduction of an infinite chain is specified by parametric equations

$$
u^{1}=u^{1}\left(R^{1}, \ldots, R^{m}\right), \quad u^{2}=u^{2}\left(R^{1}, \ldots, R^{m}\right), \quad u^{3}=u^{3}\left(R^{1}, \ldots, R^{m}\right), \ldots,
$$

etc, where the Riemann invariants $R^{1}, \ldots, R^{m}$ solve a diagonal system (8) whose characteristic speeds satisfy the semi-Hamiltonian property (9). It is required that all equations of the chain are satisfied identically modulo (8). Thus, an infinite chain reduces to a system with finitely 
many dependent variables. It was demonstrated in [17] that the Benney chain (2) possesses infinitely many $m$-component reductions of this type parametrized by $m$ arbitrary functions of a single variable. Based on this example, we give the following

Definition 3. A hydrodynamic chain from the class $C$ is said to be integrable if it possesses infinitely many $m$-component semi-Hamiltonian reductions parametrised by $m$ arbitrary functions of a single variable.

In Sect. 5 we prove our main result:

Theorem 2 The vanishing of the Haantjes tensor $H$ is the necessary condition for the integrability of hydrodynamic chains from the class $C$.

This theorem provides an easy-to-verify necessary condition for testing the integrability of hydrodynamic chains. We emphasize that the vanishing of the Haantjes tensor is not sufficient in general: one can easily construct examples of diagonalizable chains which possess infinitely many hydrodynamic reductions none of which are semi-Hamiltonian (see Sect. 5). To eliminate these pathological cases let us recall that for finite-component systems (7) there exists a simple tensor object which is responsible for the semi-Hamiltonian property [35]. This is a (1,3)-tensor $P_{k i j}^{s}$ (see the Appendix for explicit formulas in terms of the matrix $v_{j}^{i}$ ). Similarly to the Haantjes tensor $H$, the tensor $P$ is well-defined for hydrodynamic chains from the class $C$. We conclude this introduction by formulating the following

Conjecture. The vanishing of both tensors $H$ and $P$ is necessary and sufficient for the integrability of hydrodynamic chains from the class $C$.

The necessity of this conjecture (that is, the statement that the integrability implies the vanishing of both $H$ and $P$ ) is a relatively simple fact, see Sect. 5 for the proof. The sufficiency is a far more delicate property which we were not able to establish in general. We point out that the vanishing of $H$ alone is already sufficiently restrictive and implies the integrability in many particularly important cases (e.g. for conservative chains, etc).

\section{Classification of diagonalizable chains of the type (6)}

The structure of equations (6) implies that the only nonzero components of the Haantjes tensor $H_{j k}^{i}$ are the ones with $j \leq i+4, k \leq i+4$. Taking into account the skew-symmetry of the Haantjes tensor in the lower indices, this leaves ten essentially different components of the type $H_{j k}^{1}$. Equating them to zero we obtain the expressions for all of the ten second order partial derivatives of $h\left(u^{1}, u^{2}, u^{3}, u^{4}\right)$ : 


$$
\begin{aligned}
& h_{11}=\frac{2 g_{1} g_{12}-g_{2} g_{11}+2 h_{1} g_{13}}{g_{3}}, \\
& h_{12}=\frac{g_{22} g_{1}+g_{11}+g_{13} h_{2}+g_{23} h_{1}}{g_{3}}, \\
& h_{22}=\frac{g_{2} g_{22}+2 g_{12}+2 g_{23} h_{2}}{g_{3}}, \\
& h_{13}=\frac{g_{13}\left(h_{3}-g_{2}\right)+g_{23} g_{1}+g_{12} g_{3}+g_{33} h_{1}}{g_{3}}, \\
& h_{23}=g_{22}+\frac{g_{13}+h_{3} g_{23}+h_{2} g_{33}}{g_{3}}, \\
& h_{33}=2 g_{23}-\frac{g_{33}\left(g_{2}-2 h_{3}\right)}{g_{3}}, \\
& h_{14}=\frac{h_{4} g_{13}}{g_{3}}, \quad h_{24}=\frac{h_{4} g_{23}}{g_{3}}, \quad h_{34}=\frac{h_{4} g_{33}}{g_{3}}, \quad h_{44}=0 .
\end{aligned}
$$

Notice that these equations can be compactly written as

$$
d^{2} h=\frac{2}{g_{3}}\left(d h d g_{3}+d g_{1} d u^{2}+d g_{2} d g-\frac{1}{2} g_{2} d^{2} g\right)
$$

where both sides of the equality are understood as symmetric two-forms, and $d g, d h, d^{2} g, d^{2} h$ denote the first and second symmetric differentials of $g$ and $h$. The consistency conditions for the equations (13) lead to closed-form expressions for all third order partial derivatives of the function $g\left(u^{1}, u^{2}, u^{3}\right)$ in terms of its lower order derivatives:

$$
\begin{aligned}
g_{333} & =\frac{2 g_{33}^{2}}{g_{3}}, \quad g_{133}=\frac{2 g_{13} g_{33}}{g_{3}}, \quad g_{233}=\frac{2 g_{23} g_{33}}{g_{3}} \\
g_{113} & =\frac{2 g_{13}^{2}}{g_{3}}, \quad g_{123}=\frac{2 g_{13} g_{23}}{g_{3}}, \quad g_{223}=\frac{2 g_{23}^{2}}{g_{3}} \\
g_{222} & =\frac{2}{g_{3}^{2}}\left(g_{2} g_{23}^{2}+g_{23}\left(g_{3} g_{22}+2 g_{13}\right)-g_{33}\left(g_{2} g_{22}+2 g_{12}\right)\right) \\
g_{122} & =\frac{2}{g_{3}^{2}}\left(g_{1} g_{23}^{2}+g_{13}\left(g_{3} g_{22}+g_{13}\right)-g_{33}\left(g_{1} g_{22}+g_{11}\right)\right) \\
g_{112} & =\frac{2}{g_{3}^{2}}\left(g_{33}\left(g_{2} g_{11}-2 g_{1} g_{12}\right)-g_{13}\left(g_{2} g_{13}-2 g_{3} g_{12}\right)-g_{23}\left(g_{3} g_{11}-2 g_{1} g_{13}\right)\right) \\
g_{111} & =\frac{2}{g_{3}^{2}}\left(\left(g_{1}+g_{2}^{2}\right) g_{13}^{2}+g_{1}^{2} g_{23}^{2}+g_{3}^{2}\left(g_{12}^{2}-g_{11} g_{22}\right)-g_{22} g_{33} g_{1}^{2}\right. \\
& +g_{13} g_{3}\left(g_{11}+2\left(g_{1} g_{22}-g_{2} g_{12}\right)\right)+2 g_{23}\left(g_{2}\left(g_{3} g_{11}-g_{1} g_{13}\right)-g_{1} g_{3} g_{12}\right) \\
& \left.-g_{33}\left(\left(g_{1}+g_{2}^{2}\right) g_{11}-2 g_{1} g_{2} g_{12}\right)\right) .
\end{aligned}
$$

This system is in involution and its general solution depends on 10 integration constants, indeed, the values of $g$ and its partial derivatives up to the second order can be prescribed arbitrarily at any point $u_{0}^{1}, u_{0}^{2}, u_{0}^{3}$. The system (14) was first derived in [34] from the requirement that the chain (6) is embedded into a hierarchy of commuting hydrodynamic chains of the Egorov type. Exactly the same equations for $g$ were obtained in [13] by applying the method of hydrodynamic reductions to the PDE

$$
u_{t t}=g\left(u_{x x}, u_{x t}, u_{x y}\right)
$$


which is naturally associated with the chain (6); here the function $g$ is the same as in (6), (14). Thus, for hydrodynamic chains of the type (6) the condition of diagonalizability is necessary and sufficient for the integrability.

One can show that the vanishing of other components of the Haantjes tensor does not impose any additional constraints on the derivatives of $g$ and $h$. Thus, writing the fourth equation of the chain (6) in the form $u_{t}^{4}=s\left(u^{1}, u^{2}, u^{3}, u^{4}, u^{5}\right)_{x}$ and setting $H_{j k}^{2}=0$ one obtains the expressions for all second order partial derivatives of $s$ in terms of $h$ and $g$, which are analogous to (13). The consistency conditions are satisfied identically modulo (13), (14). Similarly, the condition $H_{j k}^{3}=0$ specifies the right hand side of the fifth equation of the chain, etc.

To explicitly calculate $g\left(u^{1}, u^{2}, u^{3}\right)$ we will follow [34]. The main observation is that the first six equations in (14) imply that the function $1 / g_{3}$ is linear, $1 / g_{3}=\alpha+\beta u^{1}+\gamma u^{2}+\delta u^{3}$. If $\delta \neq 0$ then, up to a linear change of variables, one can assume that $1 / g_{3}=u^{3}$. Similarly, if $\delta=0, \gamma \neq 0$, one can set $1 / g_{3}=u^{2}$. If $\delta=\gamma=0, \beta \neq 0$ one has $1 / g_{3}=u^{1}$. The last possibility is $1 / g_{3}=1$. Thus, we have four cases to consider:

$$
g=u^{3}+p\left(u^{1}, u^{2}\right), \quad g=\frac{u^{3}}{u^{1}}+p\left(u^{1}, u^{2}\right), \quad g=\frac{u^{3}}{u^{2}}+p\left(u^{1}, u^{2}\right), \quad g=\ln u^{3}+p\left(u^{1}, u^{2}\right) ;
$$

here the function $p\left(u^{1}, u^{2}\right)$ can be recovered after the substitution into the remaining four equations (14). In each of these cases the resulting equations for $p\left(u^{1}, u^{2}\right)$ integrate explicitly, see [34], leading to the four essentially different canonical forms:

$$
\begin{aligned}
g & =u^{3}+\frac{1}{4 A}\left(A u^{2}+2 B u^{1}\right)^{2}+C e^{-A u^{1}} \\
g & =\frac{u^{3}}{u^{1}}+\left(\frac{1}{u^{1}}-\frac{A}{4\left(u^{1}\right)^{2}}\right)\left(u^{2}\right)^{2}+\frac{B}{\left(u^{1}\right)^{2}} u^{2}-\frac{B^{2}}{A\left(u^{1}\right)^{2}}-C e^{-A / u^{1}}, \\
g & =\frac{u^{3}}{u^{2}}+\frac{1}{6} \eta\left(u^{1}\right)\left(u^{2}\right)^{2}, \\
g & =\ln u^{3}-\ln \sigma\left(u^{1}, u^{2}\right)-\frac{1}{4} \int \eta\left(u^{1}\right) d u^{1} .
\end{aligned}
$$

Here $\eta\left(u^{1}\right)$ is a solution to the Chazy equation [5],

$$
\eta^{\prime \prime \prime}+2 \eta \eta^{\prime \prime}-3{\eta^{\prime}}^{2}=0,
$$

and $\sigma\left(u^{1}, u^{2}\right)$ is an elliptic sigma function in the variable $u^{2}$ whose moduli are functions of $u^{1}$ expressed in terms of a solution to the Chazy equation. The details of the derivation of canonical forms (15) - (18) can be summarized as follows.

Case I. Substituting the ansatz $g=u^{3}+p\left(u^{1}, u^{2}\right)$ into (14) one arrives at the equations

$$
\begin{aligned}
& p_{111}=2\left(p_{12}^{2}-p_{11} p_{22}\right), \\
& p_{112}=p_{122}=p_{222}=0 .
\end{aligned}
$$

The last three equations imply

$$
p=\frac{1}{4} A\left(u^{2}\right)^{2}+\left(B u^{1}+D\right) u^{2}+q\left(u^{1}\right),
$$

and the substitution into the first equation results in the linear ODE $q^{\prime \prime \prime}+A q^{\prime \prime}=2 B^{2}$. Up to a transformation of the form $u^{3} \rightarrow u^{3}+\alpha u^{2}+\beta u^{1}+\gamma$ this leads to (15). 
Case II. Substituting the ansatz $g=u^{3} / u^{1}+p\left(u^{1}, u^{2}\right)$ into (14) one arrives at the equations

$$
\begin{aligned}
& p_{111}=2\left(p_{12}^{2}-p_{11} p_{22}\right)+\frac{2}{\left(u^{1}\right)^{2}}\left(p_{1}+p_{2}^{2}\right)-\frac{2}{u^{1}}\left(p_{11}+2 p_{1} p_{22}-2 p_{2} p_{12}\right), \\
& p_{112}=-\frac{2}{\left(u^{1}\right)^{2}} p_{2}-\frac{4}{u^{1}} p_{12}, \\
& p_{122}=\frac{2}{\left(u^{1}\right)^{2}}-\frac{2}{u^{1}} p_{22}, \quad p_{222}=0 .
\end{aligned}
$$

The last three equations imply

$$
p=\left(\frac{1}{u^{1}}-\frac{A}{4\left(u^{1}\right)^{2}}\right)\left(u^{2}\right)^{2}+\left(\frac{D}{u^{1}}+\frac{B}{\left(u^{1}\right)^{2}}\right) u^{2}-\frac{B^{2}}{A\left(u^{1}\right)^{2}}+q\left(u^{1}\right),
$$

and the substitution into the first equation results in the linear ODE $\left(u^{1}\right)^{3} q^{\prime \prime \prime}+u^{1}\left(6 u^{1}-A\right) q^{\prime \prime}+$ $\left(6 u^{1}-2 A\right) q^{\prime}=0$ whose basis of solutions consists of $1,1 / u^{1}$ and $e^{-A / u^{1}}$. Up to a transformation of the form $u^{3} \rightarrow u^{3}+\alpha u^{2}+\beta u^{1}+\gamma$ this implies (16). It was observed in [34] that chains from I and II are reciprocally related: under the change from $x, t$ to the new independent variables $X, T$ defined as $d X=u^{1} d x+u^{2} d t, T=t$, and the introduction of the new dependent variables $U^{1}=\frac{1}{u^{1}}, U^{2}=-\frac{u^{2}}{u^{1}}, U^{3}=-\frac{u^{3}}{u^{1}}$, etc, the chains from the case I transform to the chains from the case II, and vice versa. On the level of the corresponding equations this means that the change of variables $U^{1}=\frac{1}{u^{1}}, U^{2}=-\frac{u^{2}}{u^{1}}, P=\frac{\left(u^{2}\right)^{2}}{u^{1}}-p, G=\frac{\left(u^{2}\right)^{2}}{u^{1}}-g$ transforms the equations for $p$ from the Case I to the equations for $p$ from the Case II. Equivalently, (15) goes to (16).

Case III. Substituting the ansatz $g=u^{3} / u^{2}+p\left(u^{1}, u^{2}\right)$ into (14) one arrives at the equations

$$
\begin{aligned}
& p_{111}=\frac{2}{\left(u^{2}\right)^{2}} p_{1}^{2}+2\left(p_{12}^{2}-p_{11} p_{22}\right)+\frac{4}{u^{2}}\left(p_{1} p_{12}-p_{2} p_{11}\right), \\
& p_{112}=\frac{2}{u^{2}} p_{11}, \quad p_{122}=\frac{2}{\left(u^{2}\right)^{2}} p_{1}, \\
& p_{222}=\frac{2}{\left(u^{2}\right)^{2}} p_{2}-\frac{2}{u^{2}} p_{22} .
\end{aligned}
$$

The last three equations imply

$$
p=A+\frac{B+C u^{1}}{u^{2}}+\frac{1}{6} \eta\left(u^{1}\right)\left(u^{2}\right)^{2},
$$

and the substitution into the first relation results in the Chazy equation (19) for $\eta$. Elimination of the constants $A, B, C$ leads to (17).

Case IV. Substituting the ansatz $g=\ln u^{3}+p\left(u^{1}, u^{2}\right)$ into (14) one arrives at the equations

$$
\begin{aligned}
& p_{111}=2\left(p_{12}^{2}-p_{11} p_{22}\right)+2\left(p_{1}+p_{2}^{2}\right) p_{11}-4 p_{1} p_{2} p_{12}+2 p_{1}^{2} p_{22}, \\
& p_{112}=4 p_{1} p_{12}-2 p_{2} p_{11}, \\
& p_{122}=2 p_{11}+2 p_{1} p_{22}, \\
& p_{222}=4 p_{12}+2 p_{2} p_{22} .
\end{aligned}
$$

The general solution of the fourth equation can be represented in the form

$$
p=-\ln \sigma\left(u^{1}, u^{2}\right)-\frac{1}{4} \int \eta\left(u^{1}\right) d u^{1}
$$


here $\sigma$ solves the heat equation $4 \sigma_{1}=\sigma_{22}$ and $\eta$ is a function of $u^{1}$. It is convenient to introduce the new variable $v\left(u^{1}, u^{2}\right)$ by the formula $v=-(\ln \sigma)_{22}$. Taking into account the heat equation for $\sigma$ one has

$$
v_{1}=\frac{1}{4} v_{22}-\frac{1}{2} v^{2}+\frac{1}{2}(\ln \sigma)_{2} v_{2} .
$$

Rewritten in terms of $v$, the third equation for $p$ implies

$$
v_{22}=6 v^{2}-4 v \eta-4 \eta^{\prime},
$$

${ }^{\prime} \equiv d / d u^{1}$, the second equation is satisfied identically and the first takes the form

$$
v_{2}^{2}=4 v^{3}-4 v^{2} \eta-8 v \eta^{\prime}-\frac{8}{3} \eta^{\prime \prime}
$$

This shows that $v$ is a shift of the Weierstrass elliptic function in the variable $u^{2}$. Since $v=$ $-(\ln \sigma)_{22}$, the function $\sigma$ is the corresponding theta function. Notice that (20) can be obtained as a result of differentiation of (21) by $u^{2}$. Thus, we have two equations for $v$ :

$$
\begin{aligned}
& v_{1}=v^{2}-v \eta-\eta^{\prime}+\frac{1}{2}(\ln \sigma)_{2} v_{2}, \\
& v_{2}^{2}=4 v^{3}-4 v^{2} \eta-8 v \eta^{\prime}-\frac{8}{3} \eta^{\prime \prime} .
\end{aligned}
$$

The condition of their consistency leads to the Chazy equation for $\eta$.

\section{Classification of diagonalizable chains of the type (12)}

The condition $H_{j k}^{1}=0$ implies the following formulae for the second order partial derivatives of $h$ :

$$
\begin{aligned}
& h_{14}=\frac{h_{4} g_{13}}{g_{3}}, \quad h_{24}=\frac{h_{4} g_{23}}{g_{3}}, \quad h_{34}=\frac{h_{4} g_{33}}{g_{3}}, \quad h_{44}=0, \\
& h_{13}=-\frac{f_{22} g_{1} g_{3}}{g_{3} f_{2}}+\frac{g_{13}\left(h_{3}-g_{2}\right)+g_{23} g_{1}+g_{12} g_{3}+g_{33} h_{1}}{g_{3}}, \\
& h_{23}=g_{22}-f_{12}+\frac{\left(f_{1}-g_{2}\right) g_{3} f_{22}}{g_{3} f_{2}}+\frac{\left(h_{3}-f_{1}\right) g_{23}+f_{2} g_{13}+h_{2} g_{33}}{g_{3}}, \\
& h_{33}=2 g_{23}-\frac{f_{22} g_{3}^{2}}{g_{3} f_{2}}-\frac{g_{33}\left(f_{1}+g_{2}-2 h_{3}\right)}{g_{3}}, \\
& h_{22}=-\frac{f_{22}\left(f_{1}^{2}-2 f_{1} g_{2}+g_{2}^{2}+f_{2} g_{1}\right)}{g_{3} f_{2}}+\frac{\left(g_{2}-f_{1}\right)\left(g_{22}-2 f_{12}\right)+f_{2}\left(2 g_{12}-f_{11}\right)+2 g_{23} h_{2}}{g_{3}}, \\
& h_{12}=\frac{f_{22} g_{1}\left(f_{1}-g_{2}\right)}{g_{3} f_{2}}+\frac{g_{22} g_{1}+f_{2} g_{11}+g_{13} h_{2}+g_{23} h_{1}-2 g_{1} f_{12}}{g_{3}}, \\
& h_{11}=-\frac{g_{1}^{2} f_{22}}{g_{3} f_{2}}+\frac{2 g_{1} g_{12}+\left(f_{1}-g_{2}\right) g_{11}+2 h_{1} g_{13}-g_{1} f_{11}}{g_{3}} .
\end{aligned}
$$

By calculating the consistency conditions for the above equations we obtain the expressions for all third order partial derivatives of $g$ and $f$. 


\section{Equations for $g$ :}

$$
\begin{aligned}
& g_{333}=\frac{2 g_{33}^{2}}{g_{3}}, \quad g_{133}=\frac{2 g_{13} g_{33}}{g_{3}}, \quad g_{233}=\frac{2 g_{23} g_{33}}{g_{3}}, \\
& g_{113}=\frac{2 g_{13}^{2}}{g_{3}}, \quad g_{123}=\frac{2 g_{13} g_{23}}{g_{3}}, \quad g_{223}=\frac{2 g_{23}^{2}}{g_{3}}, \\
& g_{111}=\frac{f_{11}\left(-g_{33} g_{2} g_{1}+g_{3}\left(g_{23} g_{1}+g_{2} g_{13}-g_{3} g_{12}\right)\right)+2 f_{1}^{2}\left(g_{13}^{2}-g_{33} g_{11}\right)+f_{1} f_{11}\left(g_{33} g_{1}-g_{3} g_{13}\right)}{f_{2} g_{3}^{2}} \\
& +2 \frac{2 f_{1}\left(\left(-g_{33} g_{1}+g_{3} g_{13}\right) g_{12}+g_{23}\left(g_{1} g_{13}-g_{3} g_{11}\right)+g_{2}\left(-g_{13}^{2}+g_{33} g_{11}\right)\right)+g_{23}^{2} g_{1}^{2}}{f_{2} g_{3}^{2}} \\
& +2 \frac{g_{22}\left(-g_{1}^{2} g_{33}+2 g_{3} g_{1} g_{13}-g_{3}^{2} g_{11}\right)+g_{13}^{2}\left(g_{2}^{2}+f_{2} g_{1}\right)+g_{12}\left(2 g_{33} g_{2} g_{1}-2 g_{2} g_{3} g_{13}+g_{3}^{2} g_{12}\right)}{f_{2} g_{3}^{2}} \\
& +2 \frac{g_{11}\left(-g_{33} g_{2}^{2}-f_{2} g_{33} g_{1}+f_{2} g_{3} g_{13}\right)-2 g_{23}\left(g_{1} g_{3} g_{12}+g_{2}\left(g_{1} g_{13}-g_{3} g_{11}\right)\right)}{f_{2} g_{3}^{2}} \\
& +2 \frac{f_{12}\left(g_{33} g_{1}^{2}+g_{3}\left(-2 g_{1} g_{13}+g_{3} g_{11}\right)\right)}{f_{2} g_{3}^{2}}, \\
& g_{112}=\frac{\left(g_{33} g_{1}^{2}+g_{3}\left(g_{3} g_{11}-2 g_{1} g_{13}\right)\right) f_{22}+f_{2}\left(f_{11}\left(g_{33} g_{1}-g_{3} g_{13}\right)-2 g_{13}\left(g_{2} g_{13}-2 g_{3} g_{12}\right)\right)}{f_{2} g_{3}^{2}} \\
& -2 f_{2} \frac{g_{33}\left(2 g_{1} g_{12}-g_{2} g_{11}\right)+g_{23}\left(g_{3} g_{11}-2 g_{1} g_{13}\right)+f_{1}\left(g_{33} g_{11}-g_{13}^{2}\right)}{f_{2} g_{3}^{2}}, \\
& g_{122}=f_{22} \frac{\left(g_{1} g_{2} g_{33}+\left(g_{3} g_{13}-g_{1} g_{33}\right) f_{1}+\left(g_{3} g_{12}-g_{23} g_{1}-g_{2} g_{13}\right) g_{3}\right)}{f_{2} g_{3}^{2}} \\
& +\frac{2 f_{2}\left(g_{23}^{2} g_{1}-g_{33} g_{22} g_{1}+g_{3} g_{22} g_{13}+f_{2} g_{13}^{2}+\left(g_{33} g_{1}-g_{3} g_{13}\right) f_{12}-f_{2} g_{33} g_{11}\right)}{f_{2} g_{3}^{2}}, \\
& g_{222}=\frac{\left.\left(\left(\left(f_{1}-2 g_{2}\right) g_{33}+2 g_{3} g_{23}\right) f_{1}+\left(g_{2}^{2}+f_{2} g_{1}\right) g_{33}+\left(g_{3} g_{22}-2 g_{2} g_{23}-f_{2} g_{13}\right) g_{3}\right)\right) f_{22}}{f_{2} g_{3}^{2}} \\
& +\frac{\left(-2 f_{1}\left(f_{12} g_{33}+g_{23}^{2}-g_{33} g_{22}\right)+2\left(f_{12}\left(g_{33} g_{2}-g_{3} g_{23}\right)+g_{3} g_{23} g_{22}+g_{2}\left(g_{23}^{2}-g_{22} g_{33}\right)\right) f_{2}\right.}{f_{2} g_{3}^{2}} \\
& +\frac{f_{2}^{2}\left(g_{33}\left(f_{11}-4 g_{12}\right)+4 g_{23} g_{13}\right)}{f_{2} g_{3}^{2}} .
\end{aligned}
$$


Equations for $f$ :

$$
\begin{aligned}
& f_{111}=-\frac{f_{11}\left(g_{33}\left(f_{1}^{2}+g_{2}^{2}+f_{2} g_{1}\right)-2 g_{2} g_{3} g_{23}+g_{3}^{2} g_{22}-f_{2} g_{3} g_{13}\right)-2 f_{1} f_{11}\left(g_{33} g_{2}-g_{3} g_{23}\right)}{f_{2} g_{3}^{2}} \\
& +f_{12} \frac{g_{3}^{2} f_{11}+2\left(g_{1} g_{2} g_{33}+f_{1}\left(-g_{33} g_{1}+g_{3} g_{13}\right)+g_{3}\left(-g_{23} g_{1}-g_{2} g_{13}+g_{3} g_{12}\right)\right)}{f_{2} g_{3}^{2}} \\
& -\frac{f_{22}\left(g_{33} g_{1}^{2}-2 g_{1} g_{3} g_{13}+g_{3}^{2} g_{11}\right)}{f_{2} g_{3}^{2}}, \\
& f_{112}=\frac{f_{22} f_{11} g_{3}^{2}-f_{2}\left(f_{1} f_{11} g_{33}+f_{11}\left(g_{3} g_{23}-g_{33} g_{2}\right)+2 f_{12}\left(g_{33} g_{1}-g_{3} g_{13}\right)\right)}{f_{2} g_{3}^{2}} \\
& f_{122}=\frac{-f_{2}^{2} f_{11} g_{33}+f_{22}\left(f_{12} g_{3}^{2}+f_{2}\left(-g_{33} g_{1}+g_{3} g_{13}\right)\right)}{f_{2} g_{3}^{2}} \\
& f_{222}=\frac{f_{22}^{2} g_{3}^{2}-2 f_{2}^{2} f_{12} g_{33}+f_{2} f_{22}\left(\left(f_{1}-g_{2}\right) g_{33}+g_{3} g_{23}\right)}{f_{2} g_{3}^{2}}
\end{aligned}
$$

We have verified that the system $(22),(23)$ is in involution. Notice that the first six equations in (22) are exactly the same as in Sect. 2. Thus, there are four essentially different cases to consider.

Case I: $g=u^{3}+p\left(u^{1}, u^{2}\right)$. Substituting this ansatz into (22), (23) one arrives at the following relations:

equations for $p$ :

$$
\begin{aligned}
& p_{111}=\frac{p_{12}\left(2 p_{12}-f_{11}\right)+2 p_{11}\left(f_{12}-p_{22}\right)}{f_{2}} \\
& p_{112}=\frac{f_{22} p_{11}}{f_{2}}, \quad p_{122}=\frac{f_{22} p_{12}}{f_{2}}, \quad p_{222}=\frac{f_{22} p_{22}}{f_{2}} ;
\end{aligned}
$$

equations for $f$ :

$$
\begin{aligned}
& f_{111}=\frac{f_{11}\left(f_{12}-p_{22}\right)+2 f_{12} p_{12}-f_{22} p_{11}}{f_{2}}, \\
& f_{112}=\frac{f_{22} f_{11}}{f_{2}}, \quad f_{122}=\frac{f_{22} f_{12}}{f_{2}}, \quad f_{222}=\frac{f_{22}^{2}}{f_{2}} .
\end{aligned}
$$

The last three equations for $f$ and the last three equations for $p$ lead, up to elementary changes of variables, to the two possibilities.

Subcase $\mathrm{I}_{1}$ :

$$
f=s\left(u^{1}\right) e^{u^{2}}, \quad p=q\left(u^{1}\right) e^{u^{2}} .
$$

The substitution of these expressions into the remaining equations for $p_{111}$ and $f_{111}$ leads to a system of coupled ODEs for $s\left(u^{1}\right)$ and $q\left(u^{1}\right)$ :

$$
\begin{aligned}
q^{\prime \prime \prime} & =\frac{2\left(\left(q^{\prime}\right)^{2}-q q^{\prime \prime}\right)-q^{\prime} s^{\prime \prime}+2 q^{\prime \prime} s^{\prime}}{s}, \\
s^{\prime \prime \prime} & =\frac{s^{\prime \prime} s^{\prime}+2 s^{\prime} q^{\prime}-q s^{\prime \prime}-q^{\prime \prime} s}{s} .
\end{aligned}
$$


Setting $q=-s^{\prime}$, the second equation will be satisfied identically while the first implies a fourth order ODE $s^{\prime \prime \prime \prime} s+3\left(s^{\prime \prime}\right)^{2}-4 s^{\prime} s^{\prime \prime \prime}=0$ whose general solution is the elliptic sigma-function: $s=\sigma\left(u^{1}\right)$, here $(\ln \sigma)^{\prime \prime}=-\wp,\left(\wp^{\prime}\right)^{2}=4 \wp^{3}-c$ (notice that $\left.g_{2}=0, g_{3}=c\right)$. Thus, as a particular case we have

$$
f=\sigma\left(u^{1}\right) e^{u^{2}}, \quad p=-\sigma^{\prime}\left(u^{1}\right) e^{u^{2}} .
$$

Subcase $\mathrm{I}_{2}$ :

$$
f=\left(a u^{1}+b\right) u^{2}+s\left(u^{1}\right), \quad p=\frac{1}{2} A\left(u^{2}\right)^{2}+B u^{1} u^{2}+q\left(u^{1}\right),
$$

$a, b, A, B=$ const. The substitution of these expressions into the remaining equations for $p_{111}$ and $f_{111}$ leads to linear ODEs for $s\left(u^{1}\right)$ and $q\left(u^{1}\right)$ :

$$
\begin{aligned}
q^{\prime \prime \prime} & =\frac{2 B^{2}-B s^{\prime \prime}+2(a-A) q^{\prime \prime}}{a u^{1}+b}, \\
s^{\prime \prime \prime} & =\frac{(a-A) s^{\prime \prime}+2 a B}{a u^{1}+b} .
\end{aligned}
$$

These equations are straightforward to solve. One needs to consider two different cases: $a=$ $0, b=1$ and $a=1, b=0$. If $a=0, b=1$ then, up to unessential integration constants, we have

$$
s=\alpha e^{-A u^{1}}, \quad q=\frac{B^{2}}{2 A}\left(u^{1}\right)^{2}-\alpha \frac{B}{A} e^{-A u^{1}}+\beta e^{-2 A u^{1}} .
$$

The case $a=1, b=0$ leads to

$$
s=\frac{B}{A-1}\left(u^{1}\right)^{2}+\alpha\left(u^{1}\right)^{3-A}, \quad q=\frac{B^{2}(A-2)}{2(A-1)^{2}}\left(u^{1}\right)^{2}+\frac{\alpha B}{1-A}\left(u^{1}\right)^{3-A}+\beta\left(u^{1}\right)^{2(2-A)} .
$$

Case II: $g=u^{3} / u^{1}+p\left(u^{1}, u^{2}\right)$. Substituting this ansatz into (22), (23) one arrives at the following set of relations.

equations for $p$ :

$$
\begin{aligned}
& p_{111}=2 \frac{p_{2}^{2}+f_{1}^{2}+f_{2} p_{1}-4 p_{2} f_{1}}{f_{2} u_{1}^{2}}+\frac{\left(f_{1}-p_{2}\right)\left(f_{11}-4 p_{12}\right)+4 p_{1}\left(f_{12}-p_{22}\right)}{u_{1} f_{2}}+ \\
& \frac{p_{12}\left(2 p_{12}-f_{11}\right)+2 p_{11}\left(f_{12}-p_{22}\right)}{f_{2}}-2 \frac{p_{11}}{u_{1}} \\
& p_{112}=\frac{2}{u_{1}^{2}}\left(f_{1}-p_{2}\right)+\frac{f_{11}-4 p_{12}}{u_{1}}+\frac{2 p_{1} f_{22}}{u_{1} f_{2}}+\frac{f_{22} p_{11}}{f_{2}} \\
& p_{122}=2 \frac{f_{2}}{u_{1}^{2}}+\frac{2}{u_{1}}\left(f_{12}-p_{22}\right)+\frac{f_{22}\left(p_{2}-f_{1}\right)}{u_{1} f_{2}}+\frac{f_{22} p_{12}}{f_{2}} \\
& p_{222}=\frac{f_{22} p_{22}}{f_{2}}+\frac{f_{22}}{u_{1}}
\end{aligned}
$$

equations for $f$ :

$$
\begin{aligned}
& f_{111}=\frac{f_{11}}{f_{2}}\left(f_{12}-p_{22}-\frac{f_{2}}{u_{1}}\right)+\frac{f_{12}}{f_{2} u_{1}}\left(2 p_{2}-2 f_{1}+p_{12} u_{1}\right)-\frac{f_{22}}{f_{2} u_{1}}\left(2 p_{1}+p_{11} u_{1}\right), \\
& f_{112}=\frac{f_{11} f_{22}}{f_{2}}-2 \frac{f_{12}}{u_{1}}, \quad f_{122}=\frac{f_{12} f_{22}}{f_{2}}-\frac{f_{22}}{u_{1}}, \quad f_{222}=\frac{f_{22}^{2}}{f_{2}} .
\end{aligned}
$$


The cases I and II are reciprocally related (with thank Maxim Pavlov for pointing out at this equivalence): under the change from $x, t$ to the new independent variables $X, T$ defined as $d X=u^{1} d x+f d t, T=t$, and the introduction of the new independent variables $U^{1}=\frac{1}{u^{1}}, U^{2}=$ $-\frac{u^{2}}{u^{1}}, U^{3}=-\frac{u^{3}}{u^{1}}$, etc, the chains from the case I transform to the chains from the case II, and vice versa. On the level of the corresponding equations this means that the change of variables $U^{1}=\frac{1}{u^{1}}, U^{2}=-\frac{u^{2}}{u^{1}}, F=-\frac{f}{u^{1}}, P=\frac{u^{2}}{u^{1}} f-p$ transforms the equations for $p, f$ from the Case I to the equations for $P, F$ from the Case II. Thus, we will not discuss this case in any more detail here.

Case III: $g=u^{3} / u^{2}+p\left(u^{1}, u^{2}\right)$. Substituting this ansatz into (22), (23) one arrives at the following set of relations.

equations for $p$ :

$$
\begin{aligned}
& p_{111}=\frac{2 p_{1}^{2}}{f_{2} u_{2}^{2}}+\frac{p_{1}\left(4 p_{12}-f_{11}\right)+4 p_{11}\left(f_{1}-p_{2}\right)}{u_{2} f_{2}}+\frac{p_{12}\left(2 p_{12}-f_{11}\right)+2 p_{11}\left(f_{12}-p_{22}\right)}{f_{2}}, \\
& p_{112}=\frac{f_{22} p_{11}}{f_{2}}+2 \frac{p_{11}}{u_{2}}, \quad p_{122}=\frac{2 f_{2} p_{1}+u_{2} f_{22}\left(p_{1}+u_{2} p_{12}\right)}{f_{2} u_{2}^{2}}, \\
& p_{222}=2 \frac{f_{22}\left(p_{2}-f_{1}\right)-f_{2} p_{22}}{u_{2} f_{2}}+\frac{p_{22} f_{22}}{f_{2}}+2 \frac{f_{12}}{u^{2}}-\frac{2}{u_{2}^{2}}\left(f_{1}-p_{2}\right) ;
\end{aligned}
$$

equations for $f$ :

$$
\begin{aligned}
& f_{111}=\frac{f_{11}}{f_{2} u_{2}}\left(2\left(f_{1}-p_{2}\right)-p_{22} u_{2}\right)+\frac{f_{12}}{f_{2} u_{2}}\left(u_{2}\left(f_{11}+2 p_{12}\right)+2 p_{1}\right)-\frac{f_{22} p_{11}}{f_{2}}, \\
& f_{112}=\frac{f_{22} f_{11}}{f_{2}}+\frac{f_{11}}{u_{2}}, \quad f_{122}=\frac{f_{22} f_{12}}{f_{2}}, \quad f_{222}=\frac{f_{22}^{2}}{f_{2}}-\frac{f_{22}}{u_{2}} .
\end{aligned}
$$

The last three equations for $f$ and the last three equations for $p$ lead to the two essentially different possibilities:

Subcase $\mathrm{III}_{1}$ :

$$
f=s\left(u^{1}\right)\left(u^{2}\right)^{k}, \quad p=q\left(u^{1}\right)\left(u^{2}\right)^{k+1},
$$

$k=$ const. The substitution of these expressions into the remaining equations for $p_{111}$ and $f_{111}$ leads to the coupled system of ODEs for $s\left(u^{1}\right)$ and $q\left(u^{1}\right)$ :

$$
\begin{aligned}
q^{\prime \prime \prime} & =\frac{k+2}{k s}\left(2(k+2)\left(q^{\prime}\right)^{2}-2(k+1) q q^{\prime \prime}-q^{\prime} s^{\prime \prime}+2 q^{\prime \prime} s^{\prime}\right), \\
s^{\prime \prime \prime} & =\frac{k+2}{k s}\left(s^{\prime \prime} s^{\prime}-k(k+1) s^{\prime \prime} q+2 k s^{\prime} q^{\prime}\right)-(k-1) q^{\prime \prime} .
\end{aligned}
$$

Notice that under the substitution $s=k=1$ the equation for $q$ reduces to the Chazy equation (19) for $q\left(u^{1}\right)=\frac{1}{6} \eta\left(u^{1}\right)$.

Subcase $\mathrm{III}_{2}$ :

$$
f=\left(a u^{1}+b\right) \ln u^{2}+s\left(u^{1}\right), \quad p=q\left(u^{1}\right) u^{2}+a\left(u^{2} \ln u^{2}-u^{2}\right),
$$

$a, b=$ const. The substitution of these expressions into the remaining equations for $p_{111}$ and $f_{111}$ leads to the coupled system of ODEs for $s\left(u^{1}\right)$ and $q\left(u^{1}\right)$ :

$$
\begin{aligned}
q^{\prime \prime \prime} & =\frac{8\left(q^{\prime}\right)^{2}-4 q q^{\prime \prime}-2 q^{\prime} s^{\prime \prime}+4 q^{\prime \prime} s^{\prime}}{a u^{1}+b}, \\
s^{\prime \prime \prime} & =2 \frac{s^{\prime \prime}\left(s^{\prime}-q\right)+2 a q^{\prime}}{a u^{1}+b}+q^{\prime \prime} .
\end{aligned}
$$


Case IV: $g=\ln u^{3}+p\left(u^{1}, u^{2}\right)$. Substituting this ansatz into (22), (23) one obtains equations for $p$ :

$$
\begin{aligned}
& p_{111}=\frac{p_{22}\left(2 p_{1}^{2}-2 p_{11}\right)+p_{11}\left(p_{2}\left(2 p_{2}-4 f_{1}\right)+2\left(f_{1}^{2}+f_{12}+f_{2} p_{1}\right)\right)+p_{12}\left(2 p_{12}-f_{11}+4 p_{1}\left(f_{1}-p_{2}\right)\right)}{f_{2}} \\
& -\frac{p_{1}\left(2 p_{1} f_{12}+\left(f_{1}-p_{2}\right) f_{11}\right)}{f_{2}} \\
& p_{112}=\frac{f_{22}\left(p_{11}-p_{1}^{2}\right)}{f_{2}}+p_{1}\left(4 p_{12}-f_{11}\right)+2 p_{11}\left(f_{1}-p_{2}\right), \\
& p_{122}=\frac{f_{22}}{f_{2}}\left(p_{1}\left(f_{1}-p_{2}\right)+p_{12}\right)+2 p_{1}\left(p_{22}-f_{12}\right)+2 f_{2} p_{11}, \\
& p_{222}=\frac{f_{22}}{f_{2}}\left(p_{22}+f_{1}\left(2 p_{2}-f_{1}\right)-p_{2}^{2}\right)+2\left(p_{22}-f_{12}\right)\left(p_{2}-f_{1}\right)-f_{22} p_{1}+f_{2}\left(4 p_{12}-f_{11}\right) ;
\end{aligned}
$$

equations for $f$ :

$$
\begin{aligned}
& f_{111}=\frac{f_{11}}{f_{2}}\left(f_{1}^{2}+p_{2}^{2}+f_{2} p_{1}-p_{22}-2 f_{1} p_{2}+f_{12}\right)+2 \frac{f_{12}}{f_{2}}\left(p_{1}\left(f_{1}-p_{2}\right)+p_{12}\right)+\frac{f_{22}}{f_{2}}\left(p_{1}^{2}-p_{11}\right), \\
& f_{112}=\frac{f_{22} f_{11}}{f_{2}}+f_{11}\left(f_{1}-p_{2}\right)+2 f_{12} p_{1}, \quad f_{122}=\frac{f_{22} f_{12}}{f_{2}}+f_{2} f_{11}+f_{22} p_{1}, \\
& f_{222}=\frac{f_{22}^{2}}{f_{2}}+2 f_{2} f_{12}+f_{22}\left(p_{2}-f_{1}\right) .
\end{aligned}
$$

Although this system is in involution and reduces to the corresponding Case 4 in Sect. 2 under the substitution $f=u^{2}$, we were not able to integrate it in general. Let us just mention that the last three relations for $f$ imply the Monge-Ampere equation $f_{11} f_{22}-f_{12}^{2}=a\left(u^{1}\right) f_{2}^{2}$. This suggests a separable ansatz $f=s\left(u^{1}\right) r\left(u^{2}\right)$. A simple analysis leads to two possibilities:

(i) $f=s\left(u^{1}\right)\left(u^{2}\right)^{k}, \quad p=\frac{1}{1-k} s^{\prime}\left(u^{1}\right)\left(u^{2}\right)^{k+1}-\ln u^{2}$, where $s$ satisfies the equation $s s^{\prime \prime}-\frac{k}{k-1}\left(s^{\prime}\right)^{2}=$ 0 . Without any loss of generality one can take $s=\left(u^{1}\right)^{1-k}$.

(ii) $f=s\left(u^{1}\right) e^{u^{2}}, \quad p=-s^{\prime}\left(u^{1}\right) e^{u^{2}}$, where $s$ satisfies the equation $s s^{\prime \prime}-\left(s^{\prime}\right)^{2}=0$. Up to a linear transformation of $u^{1}$ one has $s=e^{u^{1}}$.

\section{Classification of diagonalizable Hamiltonain chains}

It was observed in $[23,24]$ that the Benney chain (2) can be represented in the Hamiltonian form

$$
\mathbf{u}_{t}=\left(B \frac{d}{d x}+\frac{d}{d x} B^{t}\right) \frac{\partial h}{\partial \mathbf{u}}
$$

where $B^{i j}=(i-1) u_{i+j-2}$ and $h=\left(u^{3}+\left(u^{1}\right)^{2}\right) / 2$ is the Hamiltonian density. Further integrable examples can be constructed by looking at Hamiltonian densities in the form $h=u^{3}+p\left(u^{1}, u^{2}\right)$ and imposing the constraint $H_{j k}^{1}=0$ on the corresponding Hamiltonian chain (24). This implies the relations

$$
p_{111}\left(2+u^{1} p_{22}\right)=p_{11} p_{22}-p_{12}^{2}, \quad p_{112}=p_{122}=p_{222}=0
$$

which, up to a natural equivalence $h \rightarrow \alpha h+a u^{1}+b u^{2}+c$, lead to Hamiltonian densities of the form

$$
h=u^{3}+\alpha\left(u^{1}\right)^{2}+\beta u^{1} u^{2}+\gamma\left(u^{2}\right)^{2}+\delta\left(u^{1}\right)^{3}
$$


where the constants $\alpha, \beta, \gamma, \delta$ satisfy a single relation $\beta^{2}-4 \alpha \gamma+12 \delta=0$. This class was introduced for the first time in [25] where it was shown that the corresponding Hamiltonian chains possess an infinity of conservation laws which Poisson commute and form a complete system. One can prove that all other components $H_{j k}^{i}, i \geq 2$, of the Haantjes tensor are identically zero.

The aim of this section is to characterize all densities of the form $h\left(u^{1}, u^{2}, u^{3}\right)$ such that the Haantjes tensor of the Hamiltonian chain (24) is zero. As in the previous Sections, the condition $H_{j k}^{1}=0$ provides expressions for all third order partial derivatives of $h$, the simplest six of them being

$$
\begin{aligned}
h_{333} & =\frac{5 h_{33}^{2}}{2 h_{3}}, \quad h_{133}=\frac{5 h_{13} h_{33}}{2 h_{3}}, \quad h_{233}=\frac{5 h_{23} h_{33}}{2 h_{3}}, \\
h_{113} & =\frac{3 h_{13}^{2}+2 h_{33} h_{11}}{2 h_{3}}, \\
h_{123} & =\frac{3 h_{13} h_{23}+2 h_{33} h_{12}}{2 h_{3}}, \\
h_{223} & =\frac{3 h_{23}^{2}+2 h_{33} h_{22}}{2 h_{3}}
\end{aligned}
$$

the remaining expressions for $h_{111}, h_{112}, h_{122}, h_{222}$ are not written out explicitly due to their complexity. We have verified that the system for $h$ is in involution and its solution space is 10-dimensional. The first three equations in (26) imply that $h_{33}=s h_{3}^{5 / 2}, s=$ const. Thus, there are 2 cases to consider.

Case I: $s=0$. In this case $h$ is linear in $u^{3}$ and the equations (26) imply

$$
h=\frac{u^{3}}{\left(c+a u^{1}+b u^{2}\right)^{2}}+p\left(u^{1}, u^{2}\right) .
$$

This ansatz can be simplified by utilizing the canonical transformations

$$
\tilde{u}^{1}=\lambda u^{1}, \quad \tilde{u}^{2}=u^{2}, \quad \tilde{u}^{3}=\frac{1}{\lambda} u^{3}, \quad \tilde{u}^{4}=\frac{1}{\lambda^{2}} u^{4}, \ldots,
$$

etc, $\lambda=$ const, and

$$
\tilde{u}^{1}=u^{1}, \quad \tilde{u}^{2}=u^{2}+s u^{1}, \quad \tilde{u}^{3}=u^{3}+2 s u^{2}+s^{2} u^{1}, \ldots,
$$

etc, $s=$ const. One can show that both transformations preserve the Poisson bracket specified by (24). Hence, they can be used to simplify the Hamiltonian. Suppose, for instance, that $b \neq 0$. Then, up to the first canonical transformation, one can assume the ansatz $h=u^{3} /\left(c+u^{2}\right)^{2}+$ $p\left(u^{1}, u^{2}\right)$. If $b=0$ then $h=u^{3} /\left(c+u^{1}\right)^{2}+p\left(u^{1}, u^{2}\right)$. Thus, there are two subcases:

Subcase $\mathrm{I}_{1}: h=u^{3} /\left(c+u^{1}\right)^{2}+p\left(u^{1}, u^{2}\right)$. The substitution of this ansatz into the four remaining equations for $h$ implies the following system for $p$ :

$$
\begin{aligned}
& p_{111}=-\frac{16\left(u^{2}\right)^{2}+\left(c+u^{1}\right)^{3}\left(8\left(u^{2}\right)^{2} p_{22}+4\left(3 c+u^{1}\right) u^{1} p_{12}\right)+2\left(c+u^{1}\right)^{4}\left(5 c+u^{1}\right) p_{11}}{c\left(c+u^{1}\right)^{5}\left(2+u^{1}\left(c+u^{1}\right)^{2} p_{22}\right)}+ \\
& \frac{\left(c+u^{1}\right)\left(c^{2} p_{12}^{2}-c\left(c-3 u^{1}\right) p_{22} p_{11}\right)}{c\left(2+u^{1}\left(c+u^{1}\right)^{2} p_{22}\right)}, \\
& p_{112}=2 \frac{2 u^{2}-c\left(c+u^{1}\right)^{3} p_{12}}{c\left(c+u^{1}\right)^{4}}, \quad p_{122}=-\frac{2+c\left(c+u^{1}\right)^{2} p_{22}}{c\left(c+u^{1}\right)^{3}}, \quad p_{222}=0 .
\end{aligned}
$$


The last three equations lead to $p\left(u^{1}, u^{2}\right)=\frac{1+\alpha c\left(c+u^{1}\right)}{c\left(c+u^{1}\right)^{2}}\left(u^{2}\right)^{2}+\frac{\beta u^{2}}{c+u^{1}}+q\left(u^{1}\right)$ where $\alpha$ and $\beta$ are arbitrary constants. The substitution into the first equation gives a linear ODE for $q$,

$$
\left(\alpha c^{2}-3 u^{1} \alpha c-4\right) q^{\prime \prime}-\left(c+u^{1}\right)\left(1+c u^{1} \alpha\right) q^{\prime \prime \prime}=\frac{c^{2} \beta^{2}}{2\left(c+u^{1}\right)^{3}} .
$$

Without any loss of generality one has

$$
q\left(u^{1}\right)=\frac{m}{\left(c+u^{1}\right)^{2}}+\frac{n}{c+u^{1}}
$$

where the constants $m, n$ satisfy a single relation $2\left(1-c^{2} \alpha\right) n-6 c \alpha m+\frac{1}{2} c^{2} \beta^{2}=0$. Ultimately, we have

$$
h=u^{3} /\left(c+u^{1}\right)^{2}+\frac{1+\alpha c\left(c+u^{1}\right)}{c\left(c+u^{1}\right)^{2}}\left(u^{2}\right)^{2}+\frac{\beta u^{2}}{c+u^{1}}+\frac{m}{\left(c+u^{1}\right)^{2}}+\frac{n}{c+u^{1}} .
$$

Notice that the constant $\beta$ can be eliminated by the second canonical transformation.

Subcase $\mathrm{I}_{2}: h=u^{3} /\left(c+u^{2}\right)^{2}+p\left(u^{1}, u^{2}\right)$. The substitution of this ansatz into the four remaining equations for $h$ implies the following system for $p$,

$$
\begin{aligned}
& p_{222}=\frac{\left(u^{1}\right)^{3}\left(c+u^{2}\right) p_{111}-2 c\left(u^{1}\right)^{2} p_{11}-2 c u^{1}\left(c-u^{2}\right) p_{12}-\left(5 c-3 u^{2}\right)\left(c-u^{2}\right)^{2} p_{22}}{\left(c-u^{2}\right)^{3}\left(c+u^{2}\right)}, \\
& p_{122}=\frac{\left(u^{1}\right)^{2}\left(c+u^{2}\right) p_{111}-2 c u^{1} p_{11}-2\left(2 c^{2}-3 c u^{2}+\left(u^{2}\right)^{2}\right) p_{12}}{\left(c-u^{2}\right)^{2}\left(c+u^{2}\right)}, \\
& p_{112}=\frac{u^{1}\left(c+u^{2}\right) p_{111}+\left(u^{2}-3 c\right) p_{11}}{\left(c-u^{2}\right)\left(c+u^{2}\right)}
\end{aligned}
$$

along with one more relation which we do not write out due to its complexity. These three relations imply $p\left(u^{1}, u^{2}\right)=\frac{u^{1} u^{2}-\alpha u^{2}-\frac{\alpha c}{3}+\left(c-u^{2}\right)^{3} g(\eta)}{\left(c+u_{2}\right)^{2}}$ where $g(\eta)$ is an arbitrary function of a single variable $\eta=\frac{u^{1}}{c-u^{2}}$. The result of the substitution of this ansatz into the remaining equation for $p$ factors into a product of two terms leading to the cases (i) and (ii) below:

(i) The function $g$ satisfies a first order ODE

$$
4 c^{2} \eta^{2} g^{\prime}-12 c^{2} \eta g-1-\alpha \eta+2 c \eta^{2}=0,
$$

the solution of this equation is $g(\eta)=\mu \eta^{3}+\frac{\eta}{4 c}-\frac{\alpha}{12 c^{2}}-\frac{1}{16 c^{2} \eta}$. This results in the Hamiltonian densities of the form

$$
h=\frac{u^{3}}{\left(c+u^{2}\right)^{2}}+\frac{u^{1} u^{2}}{\left(c+u^{2}\right)^{2}}-\alpha \frac{u^{2}+c / 3}{\left(c+u^{2}\right)^{2}}+\mu \frac{\left(u^{1}\right)^{3}}{\left(c+u^{2}\right)^{2}}+\frac{1}{4 c} \frac{\left(c-u^{2}\right)^{3}}{\left(c+u^{2}\right)^{2}}\left(\frac{u^{1}}{c-u^{2}}-\frac{\alpha}{3 c}-\frac{c-u^{2}}{4 c u^{1}}\right) .
$$

(ii) The function $g$ solves a third order ODE

$$
\left(4 c^{2} \eta^{2} g^{\prime}-12 c^{2} \eta g-1-\alpha \eta+2 c \eta^{2}\right) g^{\prime \prime \prime}+\left(\alpha-4 c \eta+12 c^{2} g+4 c^{2} \eta g^{\prime}-2 c^{2} \eta^{2} g^{\prime \prime}\right) g^{\prime \prime}+\left(4 c-8 c^{2} g^{\prime}\right) g^{\prime}-\frac{1}{2}=0 .
$$

Although this equation appears to be difficult to solve in general, the case $c=0$ leads, upon elementary integration, to Hamiltonian densities of the form

$$
h=\frac{u^{3}}{\left(u^{2}\right)^{2}}+\alpha \frac{\left(u^{1}\right)^{2}}{u^{2}}+\beta \frac{u^{1}}{u^{2}}+\gamma \frac{1}{u^{2}}+\delta \frac{\left(u^{1}\right)^{3}}{\left(u^{2}\right)^{2}}
$$


where the constants $\alpha, \beta, \gamma, \delta$ satisfy a single relation $\beta^{2}-4 \alpha \gamma+12 \delta=0$.

Remark. The apparent similarity of the cases (25) and (29) is not accidental and manifests the important reciprocal invariance of the class of Hamiltonian chains (24). Let us point out that the first two equations in (24) are conservative:

$$
\begin{aligned}
u_{t}^{1} & =\left(u^{1} h_{2}+2 u^{2} h_{3}\right)_{x}, \\
u_{t}^{2} & =\left(u^{1} h_{1}+2 u^{2} h_{2}+3 u^{3} h_{3}-h\right)_{x} .
\end{aligned}
$$

The conserved densities $u^{1}$ and $u^{2}$ play the roles of a Casimir and momentum of the Poisson bracket (24), respectively. Let us change from $x, t$ to the new independent variables $X, T$ where

$$
d X=u^{2} d x+\left(u^{1} h_{1}+2 u^{2} h_{2}+3 u^{3} h_{3}-h\right) d t, \quad T=t .
$$

It is known that reciprocal transformations of this type preserve Poisson brackets of the form (24), e.g. [8] (it is crucial that $u^{2}$ is the momentum of the corresponding Poisson bracket). One can verify directly that performing the above change of independent variables and introducing

$$
H=\frac{h}{u^{2}}, \quad U^{1}=\frac{u^{1}}{u^{2}}, \quad U^{2}=\frac{1}{u^{2}}, \quad U^{3}=\frac{u^{3}}{\left(u^{2}\right)^{3}}, \quad \ldots, \quad U^{n}=\frac{u^{n}}{\left(u^{2}\right)^{n}},
$$

one arrives at the system which takes the original form (24) in the variables $X, T, U^{n}, H$. Thus, the above reciprocal transformation is canonical. One can verify that the change of variables (30) identifies (25) and (29).

Case II: $s \neq 0$. In this case the elementary integration gives

$$
h=\left(\gamma u^{3}+p\left(u^{1}, u^{2}\right)\right)^{1 / 3}+q\left(u^{1}, u^{2}\right),
$$

and the substitution into the last three equations (26) implies that $q$ is linear. Up to the equivalence $h \rightarrow \alpha h+a u^{1}+b u^{2}+c$ we thus have

$$
h=\left(u^{3}+p\left(u^{1}, u^{2}\right)\right)^{1 / 3} .
$$

The substitution of this ansatz into the remaining equations for $h$ implies a complicated system of third order PDEs for $p\left(u^{1}, u^{2}\right)$. A useful observation is that this system is invariant under a 3-parameter group of point symmetries which is generated by the two canonical transformations (27), (28) and the reciprocal transformation (30). The infinitesimal generators of these symmetries are

$$
X_{1}=u^{1} \partial_{u^{1}}-p \partial_{p}, \quad X_{2}=u^{1} \partial_{u^{2}}-2 u^{2} \partial_{p}, \quad X_{3}=\left(u^{1}\right)^{2} \partial_{u^{1}}+u^{1} u^{2} \partial_{u^{2}}+\left(3 p u^{1}+2\left(u^{2}\right)^{2}\right) \partial_{p} ;
$$

they satisfy the commutator relations

$$
\left[X_{1}, X_{2}\right]=X_{2}, \quad\left[X_{1}, X_{3}\right]=X_{3}, \quad\left[X_{2}, X_{3}\right]=0 .
$$

These symmetries suggest a change of variables which simplifies the equations for $p$. The idea is to choose new coordinates such that the symmetry generators assume the simplest possible form. Introducing $\xi=-\frac{1}{u^{1}}, \eta=\frac{u^{2}}{u^{1}}, s=\frac{p u^{1}+\left(u^{2}\right)^{2}}{\left(u^{1}\right)^{4}}$, we have

$$
X_{1}=-\xi \partial_{\xi}-\eta \partial_{\eta}-4 s \partial_{s}, \quad X_{2}=\partial_{\eta}, \quad X_{3}=\partial_{\xi}
$$


Setting $s=s(\xi, \eta)$ we have $p=s(\xi, \eta)\left(u^{1}\right)^{3}-\frac{\left(u^{2}\right)^{2}}{u^{1}}$. In terms of $s(\xi, \eta)$ the equations for $p$ assume a remarkable symmetric form

$$
\begin{aligned}
& s_{\xi \xi \eta} s_{\eta \eta}-s_{\eta \eta \eta} s_{\xi \xi}=4 s_{\eta}, \\
& s_{\xi \eta \eta} s_{\xi \xi}-s_{\xi \xi \xi} s_{\eta \eta}=4 s_{\xi}, \\
& s_{\eta \eta \eta} s_{\xi}+s_{\xi \eta \eta} s_{\eta}=s_{\eta \eta} s_{\xi \eta}, \\
& s_{\xi \xi \xi} s_{\eta}+s_{\xi \xi \eta} s_{\xi}=s_{\xi \xi} s_{\xi \eta}, \\
& s_{\eta \eta \eta} s_{\xi \xi} s_{\eta}+s_{\xi \eta \eta} s_{\eta \eta} s_{\xi}+4 s_{\eta}^{2}=\frac{1}{2} s_{\eta \eta}\left(12 s+s_{\xi \eta}^{2}+s_{\xi \xi} s_{\eta \eta}\right), \\
& s_{\xi \xi \xi} s_{\eta \eta} s_{\xi}+s_{\xi \xi \eta} s_{\xi \xi} s_{\eta}+4 s_{\xi}^{2}=\frac{1}{2} s_{\xi \xi}\left(12 s+s_{\xi \eta}^{2}+s_{\xi \xi} s_{\eta \eta}\right)
\end{aligned}
$$

in the process of derivation of these equations we have assumed that $s_{\xi}, s_{\eta}$ and $s_{\xi \xi} s_{\eta}^{2}-s_{\eta \eta} s_{\xi}^{2}$ are nonzero: these expressions appear as denominators in the intermediate calculations. Particular cases when some of these expressions vanish will be discussed below.

To solve the equations (31) - (36) we proceed as follows. Differentiating (33), (34) and using (31), (32) we obtain four relations among the fourth order derivatives of $s$,

$$
\begin{aligned}
& s_{\xi} s_{\eta \eta \eta \eta}+s_{\eta} s_{\xi \eta \eta \eta}=0, \quad s_{\xi} s_{\xi \eta \eta \eta}+s_{\eta}\left(s_{\xi \xi \eta \eta}-4\right)=0, \\
& s_{\xi}\left(s_{\xi \xi \eta \eta}-4\right)+s_{\eta} s_{\xi \xi \xi \eta}=0, \quad s_{\xi} s_{\xi \xi \xi \eta}+s_{\eta} s_{\xi \xi \xi \xi}=0,
\end{aligned}
$$

which can be parametrized as

$$
s_{\eta \eta \eta \eta}=q, \quad s_{\xi \eta \eta \eta}=q r, \quad s_{\xi \xi \eta \eta}-4=q r^{2}, \quad s_{\xi \xi \xi \eta}=q r^{3}, \quad s_{\xi \xi \xi \xi}=q r^{4},
$$

$r=-s_{\xi} / s_{\eta}$. The further analysis leads to two possibilities.

Subcase $\mathrm{II}_{1}: q=0$. In this case $s(\xi, \eta)$ is a polynomial of the form

$$
s=\xi^{2} \eta^{2}+a \xi^{3}+b \xi^{2} \eta+c \xi \eta^{2}+d \eta^{3}+\alpha \xi^{2}+\beta \xi \eta+\gamma \eta^{2}+\mu \xi+\nu \eta+\epsilon .
$$

The substitution into the remaining equations for $s$ implies the following relations among the coefficients:

$$
\beta=b c-9 a d, \quad \mu=\alpha c-3 a \gamma, \quad \nu=b \gamma-3 \alpha d, \quad 12 \epsilon+\beta^{2}+4 \alpha \gamma=4(b \nu+c \mu) .
$$

Notice that the corresponding $p\left(u^{1}, u^{2}\right)=s\left(-\frac{1}{u^{1}}, \frac{u^{2}}{u^{1}}\right)\left(u^{1}\right)^{3}-\frac{\left(u^{2}\right)^{2}}{u^{1}}$ will be a cubic polynomial in $u^{1}, u^{2}$. A particular example from this class with the Hamiltonian density $h=\left(u^{3}+\tau\right)^{1 / 3}$ was discussed in [25]. It corresponds to the case where $a=-\tau$ and all other coefficients of $s$ are zero.

Subcase $\mathrm{II}_{2}: q \neq 0$. Then the consistency conditions of (37) imply the relations $q_{\xi}=(q r)_{\eta}, r_{\xi}=$ $r r_{\eta}$. Taking into account that $r=-s_{\xi} / s_{\eta}$ we have $s_{\xi \xi} s_{\eta}^{2}-s_{\eta \eta} s_{\xi}^{2}=0$. This case is discussed below.

There are 3 more possibilities we need to consider to complete the classification (notice that the equations (31) - (36) can no longer be used since they we derived under the assumption that certain expressions are not vanishing).

Subcase $\mathrm{II}_{3}: s_{\xi}=0$ or $s_{\eta}=0$. A simple analysis leads to Hamiltonian densities of the form

$$
h=\left(u^{3}+\alpha\left(u^{1}\right)^{3}-\frac{\left(u^{2}\right)^{2}}{u^{1}}\right)^{1 / 3},
$$


$\alpha=$ const.

Subcase $\mathrm{II}_{4}: s_{\xi \xi} s_{\eta}^{2}-s_{\eta \eta} s_{\xi}^{2}=0$. Setting $r=-\frac{s_{\xi}}{s_{\eta}}$ one obtains

$$
r_{\xi}=r r_{\eta}, \quad s_{\xi}=-r s_{\eta} .
$$

Calculating partial derivatives of $s$ with the help of the above relations and substituting them into the conditions $H_{j k}^{1}=0$ we obtain $r_{\eta \eta}=0$; without any loss of generality one can set $r=-\frac{\eta}{\xi}$. This implies $\xi s_{\xi}-\eta s_{\eta}=0$, therefore, $s=s(z), z=\xi \eta$. For $s(z)$ we obtain the ODE

$$
8 z^{2} s^{\prime \prime \prime} s^{\prime}+8 z s^{\prime \prime} s^{\prime}-4 z^{2}\left(s^{\prime \prime}\right)^{2}-\left(s^{\prime}\right)^{2}-12 s=0 .
$$

A particular solution $s=z^{2}+\alpha(-z)^{3 / 2}$ leads to the Hamiltonian density

$$
h=\left(u^{3}+\alpha\left(u^{2}\right)^{3 / 2}\right)^{1 / 3},
$$

$\alpha=$ const.

\section{Hydrodynamic reductions and the diagonalizability}

To illustrate the method of hydrodynamic reductions we consider the Benney chain (2),

$$
\begin{aligned}
& u_{t}^{1}=u_{x}^{2}, \\
& u_{t}^{2}=u_{x}^{3}+u^{1} u_{x}^{1}, \\
& u_{t}^{3}=u_{x}^{4}+2 u^{2} u_{x}^{1}, \\
& u_{t}^{4}=u_{x}^{5}+3 u^{3} u_{x}^{1},
\end{aligned}
$$

etc. Following the approach of $[17,18]$ let us seek solutions in the form $u^{i}=u^{i}\left(R^{1}, \ldots, R^{m}\right)$ where the Riemann invariants $R^{1}, \ldots, R^{m}$ solve a diagonal system

$$
R_{t}^{i}=\lambda^{i}(R) R_{x}^{i}
$$

Substituting this ansatz into the Benney equations and equating to zero coefficients at $R_{x}^{i}$ we arrive at the following relations:

$$
\begin{aligned}
\partial_{i} u^{2} & =\lambda^{i} \partial_{i} u \\
\partial_{i} u^{3} & =\left(\left(\lambda^{i}\right)^{2}-u\right) \partial_{i} u \\
\partial_{i} u^{4} & =\left(\left(\lambda^{i}\right)^{3}-u \lambda^{i}-2 u^{2}\right) \partial_{i} u \\
\partial_{i} u^{5} & =\left(\left(\lambda^{i}\right)^{4}-u\left(\lambda^{i}\right)^{2}-2 u^{2} \lambda^{i}-3 u^{3}\right) \partial_{i} u
\end{aligned}
$$

etc. Here $u=u^{1}, \partial_{i}=\partial_{R^{i}}, i=1, \ldots, m$ (no summation!) The consistency conditions of the first three relations (38)-(40) imply

$$
\begin{gathered}
\partial_{i} \partial_{j} u=\frac{\partial_{j} \lambda^{i}}{\lambda^{j}-\lambda^{i}} \partial_{i} u+\frac{\partial_{i} \lambda^{j}}{\lambda^{i}-\lambda^{j}} \partial_{j} u, \\
\partial_{j} \lambda^{i} \partial_{i} u+\partial_{i} \lambda^{j} \partial_{j} u=0, \\
\lambda^{i} \partial_{j} \lambda^{i} \partial_{i} u+\lambda^{j} \partial_{i} \lambda^{j} \partial_{j} u+\partial_{i} u \partial_{j} u=0,
\end{gathered}
$$


respectively. Solving these equations for $\partial_{j} \lambda^{i}$ we arrive at the Gibbons-Tsarev system

$$
\partial_{j} \lambda^{i}=\frac{\partial_{j} u}{\lambda^{j}-\lambda^{i}}, \quad \partial_{i} \partial_{j} u=2 \frac{\partial_{i} u \partial_{j} u}{\left(\lambda^{i}-\lambda^{j}\right)^{2}} .
$$

It is a truly remarkable fact that all other consistency conditions (e.g. of the relation (41), etc), are satisfied identically modulo (42). Moreover, the semi-Hamiltonian property (9) is also automatically satisfied. Thus, the system (42) governs $m$-component reductions of the Benney chain. Up to reparametrizations $R^{i} \rightarrow f^{i}\left(R^{i}\right)$ these reductions depend on $m$ arbitrary functions of a single variable. Solutions arising within this approach are known as multiple waves, or nonlinear interactions of planar simple waves.

The above approach clearly applies to any hydrodynamic chain from the class $C$. Let us restrict, for instance, to the chains of the type (6). Looking for solutions in the form $u^{i}=$ $u^{i}\left(R^{1}, \ldots, R^{m}\right)$ and substituting this ansatz into (6) we arrive at an infinite system of relations similar to (38)-(41). The first three of them imply the 'generalized Gibbons-Tsarev system' of the form

$$
\partial_{j} \lambda^{i}=(\ldots) \partial_{j} u, \quad \partial_{i} \partial_{j} u=(\ldots) \partial_{i} u \partial_{j} u
$$

$u=u^{1}$, where dots denote complicated expression which are rational in $\lambda^{i}$ with the coefficients depending on the chain under study (that is, on $g, h$, etc). Requiring that all other consistency conditions, as well as the semi-Hamiltonian property, are satisfied identically modulo the generalized Gibbons-Tsarev system we obtain constraints on the matrix $V(u)$, see $[9,10,11,12,13,14]$ where a similar approach was applied to the classification of integrable multi-dimensional quasilinear systems. The main result of this section is the proof of the following theorem formulated in the introduction:

Theorem 2 The vanishing of the Haantjes tensor is the necessary condition for the existence of infinitely many hydrodynamic reductions and, thus, for the integrability of a hydrodynamic chain.

We will give two different proofs of this statement. Based on essentially different ideas, they may be of interest in their own right.

\section{First Proof:}

This proof is computational. Writing down the equations of the chain in the form $u_{t}^{m}=V_{n}^{m} u_{x}^{n}$ and substituting the ansatz $u^{i}=u^{i}\left(R^{1}, \ldots, R^{m}\right)$ we arrive at the infinite set of relations

$$
V_{n}^{m} \partial_{i} u^{n}=\lambda^{i} \partial_{i} u^{m}
$$

we point out that all summations here and below involve finitely many nonzero terms. Applying the operator $\partial_{j}, j \neq i$, we obtain

$$
V_{n, k}^{m} \partial_{i} u^{n} \partial_{j} u^{k}+V_{n}^{m} \partial_{i} \partial_{j} u^{n}=\partial_{j} \lambda^{i} \partial_{i} u^{m}+\lambda^{i} \partial_{i} \partial_{j} u^{m} .
$$

Interchanging the indices $i$ and $j$ and subtracting the results we arrive at the expression for $\partial_{i} \partial_{j} u^{m}$ in the form

$$
\partial_{i} \partial_{j} u^{m}=\frac{\partial_{j} \lambda^{i}}{\lambda^{j}-\lambda^{i}} \partial_{i} u^{m}+\frac{\partial_{i} \lambda^{j}}{\lambda^{i}-\lambda^{j}} \partial_{j} u^{m}+\frac{V_{n, k}^{m}-V_{k, n}^{m}}{\lambda^{i}-\lambda^{j}} \partial_{i} u^{n} \partial_{j} u^{k} .
$$

Substituting this back into (43) we arrive at a simple relation

$$
\partial_{j} \lambda^{i} \partial_{i} u^{m}+\partial_{i} \lambda^{j} \partial_{j} u^{m}=\frac{N_{n k}^{m} \partial_{i} u^{n} \partial_{j} u^{k}}{\lambda^{i}-\lambda^{j}}
$$


where $N$ is the Nijenhuis tensor of $V$. This can be rewritten in the invariant form

$$
\partial_{j} \lambda^{i} \partial_{i} \mathbf{u}+\partial_{i} \lambda^{j} \partial_{j} \mathbf{u}=\frac{N\left(\partial_{i} \mathbf{u}, \partial_{j} \mathbf{u}\right)}{\lambda^{i}-\lambda^{j}}
$$

which implies the following four relations:

$$
\begin{aligned}
& \left(\lambda^{i}\right)^{2} \partial_{j} \lambda^{i} \partial_{i} \mathbf{u}+\left(\lambda^{j}\right)^{2} \partial_{i} \lambda^{j} \partial_{j} \mathbf{u}=\frac{V^{2} N\left(\partial_{i} \mathbf{u}, \partial_{j} \mathbf{u}\right)}{\lambda^{i}-\lambda^{j}} \\
& \left(\lambda^{i}\right)^{2} \partial_{j} \lambda^{i} \partial_{i} \mathbf{u}+\lambda^{i} \lambda^{j} \partial_{i} \lambda^{j} \partial_{j} \mathbf{u}=\frac{V N\left(V \partial_{i} \mathbf{u}, \partial_{j} \mathbf{u}\right)}{\lambda^{i}-\lambda^{j}} \\
& \lambda^{i} \lambda^{j} \partial_{j} \lambda^{i} \partial_{i} \mathbf{u}+\left(\lambda^{j}\right)^{2} \partial_{i} \lambda^{j} \partial_{j} \mathbf{u}=\frac{V N\left(\partial_{i} \mathbf{u}, V \partial_{j} \mathbf{u}\right)}{\lambda^{i}-\lambda^{j}} \\
& \lambda^{i} \lambda^{j} \partial_{j} \lambda^{i} \partial_{i} \mathbf{u}+\lambda^{i} \lambda^{j} \partial_{i} \lambda^{j} \partial_{j} \mathbf{u}=\frac{N\left(V \partial_{i} \mathbf{u}, V \partial_{j} \mathbf{u}\right)}{\lambda^{i}-\lambda^{j}}
\end{aligned}
$$

For instance, the first relation can be obtained by applying the operator $V^{2}$ to (44) and using $V \partial_{i} \mathbf{u}=\lambda^{i} \partial_{i} \mathbf{u}$. Thus,

$$
V^{2} N\left(\partial_{i} \mathbf{u}, \partial_{j} \mathbf{u}\right)-V N\left(V \partial_{i} \mathbf{u}, \partial_{j} \mathbf{u}\right)-V N\left(\partial_{i} \mathbf{u}, V \partial_{j} \mathbf{u}\right)+N\left(V \partial_{i} \mathbf{u}, V \partial_{j} \mathbf{u}\right)=0 .
$$

The last relation can be rewritten in the form $H\left(\partial_{i} \mathbf{u}, \partial_{j} \mathbf{u}\right)=0$ where $H$ is the Haantjes tensor, indeed, the coordinate-free form of the relation (11) is

$$
H(X, Y)=V^{2} N(X, Y)-V N(V X, Y)-V N(X, V Y)+N(V X, V Y)
$$

where $X, Y$ are arbitrary vector fields. Assuming that the formal eigenvectors of the matrix $V$ span the space of dependent variables $\mathbf{u}$ (this is true for all examples discussed in this paper), we obtain $H=0$.

Notice that we have proved a more general result, namely, that the existence of sufficiently many two-component reductions already implies the vanishing of the Haantjes tensor $H$ (indeed, nothing changes in the proof if we set $i=1, j=2$ in the formula (44)).

\section{Second Proof:}

Our first remark is that for the chains from the class $\mathrm{C}$ one needs to know only finitely many rows of the matrix $V(u)$ to calculate each particular component of the Haantjes tensor. Let us fix the values of indices $i, j, k$ and denote by $C(i, j, k)$ the maximal number of rows needed to calculate $H_{j k}^{i}$ (counting from the first row). We need to show that $H_{j k}^{i}=0$. Let us consider an $m$-component diagonal reduction $u^{i}\left(R^{1}, \ldots, R^{m}\right), i=1,2, \ldots$ Choosing the first $m$ variables $u^{1}, \ldots, u^{m}$ as independent, we can represent the reduction in an explicit form as

$$
u^{m+1}=u^{m+1}\left(u^{1}, \ldots, u^{m}\right), \quad u^{m+2}=u^{m+2}\left(u^{1}, \ldots, u^{m}\right), \ldots,
$$

etc. Substituting these expressions into the first $m$ equations of the chain we obtain an $m$ component system $S_{m}$ for $u^{1}, \ldots, u^{m}$, while the remaining equations will be satisfied identically (by definition of reduction). Notice that the Haantjes tensor of the reduced system $S_{m}$ is identically zero since the reduction is diagonalizable. Moreover, let us choose the number $m$ sufficiently large so that the first $C(i, j, k)$ equations of the chain do not contain variables $u^{m+1}, u^{m+2}, \ldots$ (one can always do so since any equation of the chain depends on finitely many $u$ 's, and $m$ can be arbitrarily large). Then the first $C(i, j, k)$ equations of the reduced system $S_{m}$ will be 
identical to the first $C(i, j, k)$ equations of the original infinite chain. Hence, the corresponding components $H_{j k}^{i}$ for the reduced system and for the infinite chain will also be the same. This proves that all components of the Haantjes tensor of the chain are zero.

A straightforward modification of the second proof allows one to show that the existence of an infinity of semi-Hamiltonian reductions implies the vanishing of the tensor $P$. This establishes the necessity of the conjecture formulated in the Introduction.

We emphasize that the condition of diagonalizability alone is not sufficient for the integrability in general. This can be seen as follows.

Example. Let us consider the chain

$$
\begin{aligned}
& u_{t}^{1}=u_{x}^{2}+p\left(u^{1}\right) u_{x}^{1}, \\
& u_{t}^{2}=u_{x}^{3}+p\left(u^{1}\right) u_{x}^{2}+u^{1} u_{x}^{1}, \\
& u_{t}^{3}=u_{x}^{4}+p\left(u^{1}\right) u_{x}^{3}+2 u^{2} u_{x}^{1}, \\
& u_{t}^{4}=u_{x}^{5}+p\left(u^{1}\right) u_{x}^{4}+3 u^{3} u_{x}^{1},
\end{aligned}
$$

etc, which is obtained from the Benney chain $\mathbf{u}_{t}=V(\mathbf{u}) \mathbf{u}_{x}$ by the transformation $V \rightarrow V+$ $p\left(u^{1}\right) E$ where $E$ is the infinite identity matrix and $p$ is a function of $u^{1}$. One can verify that the corresponding Haantjes tensor is zero (which is not at all surprising, for instance, for finite matrices the addition of a multiple of the identity does not effect the diagonalizability). A simple calculation shows that hydrodynamic reductions of this chain are governed by exactly the same equations as in the Benney case, the only difference is that now the Riemann invariants $R^{i}$ solve the equations

$$
R_{t}^{i}=\left(\lambda^{i}(R)+p\left(u^{1}\right)\right) R_{x}^{i} .
$$

The semi-Hamiltonian property is satisfied if and only if $p^{\prime \prime}=0$. Thus, we have constructed examples which possess infinitely many hydrodynamic reductions none of which is semi-Hamiltonian.

\section{Conclusion}

We have proposed a simple and easy-to-verify necessary condition for the integrability of hydrodynamic chains based on the calculation of the Haantjes tensor. Conservative and Hamiltonian chains are considered, illustrating the general approach. We conjecture that all examples arising in Sect. 3 and 4 from the requirement of the vanishing of the Haantjes tensor (and, thus, satisfying the necessary condition for integrability) also satisfy the following properties:

(i) they possess infinitely many $m$-component hydrodynamic reductions parametrized by $m$ arbitrary functions of a single variable (thus, they are integrable in the sense of Definition 3);

(ii) they belong to infinite hierarchies of commuting hydrodynamic chains; in the Hamiltonian case they automatically possess complete systems of conservation laws which Poisson commute.

Our method leads to an abundance of new examples of hydrodynamic chains which require a further detailed investigation. We hope to address these issues in the future.

\section{Appendix: invariant formulation of the semi-Hamiltonian prop- erty}

For $m$-component systems (7) there exists a tensor object which is responsible for the semiHamiltonian property. First of all one computes the (1,3)-tensors $M$ and $K$,

$$
M_{k i j}^{s}=N_{k p}^{s} v_{q}^{p} N_{i j}^{q}+N_{p q}^{s} v_{k}^{p} N_{i j}^{q}-N_{p q}^{s} N_{i k}^{p} v_{j}^{q}-N_{p q}^{s} N_{k j}^{p} v_{i}^{q}-N_{k p}^{s} N_{i q}^{p} v_{j}^{q}-N_{k p}^{s} N_{q j}^{p} v_{i}^{q}
$$


and

$$
\begin{gathered}
K_{k i j}^{s}=b_{p}^{s} \partial_{u^{k}} N_{i j}^{p}-b_{k}^{p} \partial_{u^{p}} N_{i j}^{s}+N_{i j}^{p} \partial_{u^{p}} b_{k}^{s}-N_{k p}^{s} \partial_{u^{i}} b_{j}^{p}+N_{k p}^{s} \partial_{u^{j}} b_{i}^{p} \\
+b_{p}^{s} \partial_{u^{i}} N_{j k}^{p}-b_{i}^{p} \partial_{u^{p}} N_{j k}^{s}+N_{j k}^{p} \partial_{u^{p}} b_{i}^{s}-N_{i p}^{s} \partial_{u^{j}} b_{k}^{p}+N_{i p}^{s} \partial_{u^{k}} b_{j}^{p} \\
+b_{p}^{s} \partial_{u^{j}} N_{k i}^{p}-b_{j}^{p} \partial_{u^{p}} N_{k i}^{s}+N_{k i}^{p} \partial_{u^{p}} b_{j}^{s}-N_{j p}^{s} \partial_{u^{k}} b_{i}^{p}+N_{j p}^{s} \partial_{u^{i}} b_{k}^{p}
\end{gathered}
$$

here $b=v^{2}$, that is, $b_{j}^{i}=v_{p}^{i} v_{j}^{p}$. Using $M$ and $K$ one defines the $(1,3)$-tensor $Q$ as

$$
\begin{gathered}
Q_{k i j}^{s}=v_{k}^{p} K_{p q j}^{s} v_{i}^{q}+v_{k}^{p} K_{p i q}^{s} v_{j}^{q}-v_{q}^{p} v_{k}^{q} K_{p i j}^{s}-K_{k p q}^{s} v_{i}^{p} v_{j}^{q} \\
+4 v_{k}^{p} M_{p i j}^{s}-2 M_{k p j}^{s} v_{i}^{p}-2 M_{k i p}^{s} v_{j}^{p} .
\end{gathered}
$$

Ultimately, one introduces the tensor $P$,

$$
P_{k i j}^{s}=v_{p}^{s} Q_{k q j}^{p} v_{i}^{q}+v_{p}^{s} Q_{k i q}^{p} v_{j}^{q}-v_{q}^{s} v_{p}^{q} Q_{k i j}^{p}-Q_{k p q}^{s} v_{i}^{p} v_{j}^{q} .
$$

Theorem 3 [35] A diagonalizable system (7) is semi-Hamiltonian if and only if the tensor $P$ vanishes identically.

All these tensors can be calculated using computer algebra. Notice that they are well-defined for hydrodynamic chains of the class $C$ : all tensor operations will involve finite summations only. The invariant coordinate-free definitions of the above tensors can be found in [35].

\section{Acknowledgements}

We thank Karima Khusnutdinova, Maxim Pavlov and John Gibbons for their help and clarifying discussions.

\section{References}

[1] S.J. Alber, Associated integrable systems, J. Math. Phys. 32 (1991) 916-922.

[2] D.J. Benney, Some properties of long nonlinear waves, Stud. Appl. Math. 52 (1973) 45-50.

[3] M. Blaszak, Classical $R$-matrices on Poisson algebras and related dispersionless systems. Phys. Lett. A 297, no. 3-4 (2002) 191-195.

[4] V.M. Bukhshtaber, D.V. Leikin and M.V. Pavlov, Egorov hydrodynamic chains, the Chazy equation, and the group SL(2,C), Funktsional. Anal. i Prilozhen. 37, no. 4 (2003) 13-26.

[5] J. Chazy, Sur les équations différentielles dont l'intégrale générale poss ède un coupure essentielle mobile, C.R. Acad. Sci. Paris 150 (1910) 456-458.

[6] B.A. Dubrovin and S.P. Novikov, Hydrodynamics of weakly deformed soliton lattices: differential geometry and Hamiltonian theory, Russian Math. Surveys, 44 , no. 6 (1989) 35-124.

[7] B.A. Dubrovin, Geometry of 2D topological field theories, Lect. Notes in Math. 1620, Springer-Verlag (1996) 120-348. 
[8] E.V. Ferapontov, Nonlocal Hamiltonian operators of hydrodynamic type: differential geometry and applications, Amer. Math. Soc. Transl. (2), 170 (1995) 33-58.

[9] E.V. Ferapontov and K.R. Khusnutdinova, On integrability of (2+1)-dimensional quasilinear systems, Comm. Math. Phys. 248 (2004) 187-206; arXiv:nlin.SI/0305044.

[10] E.V. Ferapontov and K.R. Khusnutdinova, The characterization of two-component $(2+1)-$ dimensional integrable systems of hydrodynamic type, J. Phys. A: Math. Gen. 37, N8 (2004) 2949 - 2963; arXiv:nlin.SI/0310021.

[11] E.V. Ferapontov and K.R. Khusnutdinova, Hydrodynamic reductions of multi-dimensional dispersionless PDEs: the test for integrability, J. Math. Phys. 45, N6 (2004) 2365-2377; arXiv:nlin.SI/0312015.

[12] E.V. Ferapontov and K.R. Khusnutdinova, Double waves in multi-dimensional systems of hydrodynamic type: the necessary condition for integrability, arXiv:nlin.SI/0412064, submitted to Proc. Royal Soc. A (2005).

[13] E.V. Ferapontov, K.R. Khusnutdinova and M.V. Pavlov, Classification of integrable (2+1)dimensional quasilinear hierarchies, Theor. Math. Phys. (2005), to appear.

[14] E.V. Ferapontov, K.R. Khusnutdinova and S.P. Tsarev, On a class of three-dimensional integrable Lagrangians, to appear in Comm. Math. Phys. (2005); arXiv:nlin.SI/0407035.

[15] J. Gibbons, Collisionless Boltzmann equations and integrable moment equations, Physica 3D (1981) 503-511.

[16] J. Gibbons and Y. Kodama, A method for solving the dispersionless KP hierarchy and its exact solutions. II, Phys. Lett. A 135, no. 3 (1989) 167-170.

[17] J. Gibbons and S. P. Tsarev, Reductions of the Benney equations, Phys. Lett. A 211 (1996) $19-24$

[18] J. Gibbons and S. P. Tsarev, Conformal maps and reductions of the Benney equations, Phys. Lett. A 258 (1999) 263-271.

[19] J. Haantjes, On $X_{m}$-forming sets of eigenvectors, Indagationes Mathematicae 17 (1955) 158-162.

[20] K.R. Khusnutdinova and S.P. Tsarev, private communication.

[21] B.G. Konopelchenko and L. Martinez Alonso, Dispersionless scalar integrable hierarchies, Whitham hierarchy, and the quasiclassical $\bar{\partial}$-dressing method, J. Math. Phys. 43, no. 7 (2002) 3807-3823.

[22] B.G. Konopelchenko, L. Martinez Alonso and O. Ragnisco, The $\bar{\partial}$-approach to the dispersionless KP hierarchy, J. Phys. A 34, no. 47 (2001) 10209-10217.

[23] B.A. Kupershmidt and Yu.I. Manin, Long wave equations with a free surface. I. Conservation laws and solutions. (Russian) Funktsional. Anal. i Prilozhen. 11, no. 3 (1977) 31-42. 
[24] B.A. Kupershmidt and Yu.I. Manin, Long wave equations with a free surface. II. The Hamiltonian structure and the higher equations. (Russian) Funktsional. Anal. i Prilozhen. 12, no. 1 (1978) 25-37.

[25] B.A. Kupershmidt, Deformations of integrable systems. Proc. Roy. Irish Acad. Sect. A 83, no. 1 (1983) 45-74.

[26] B.A. Kupershmidt, The quasiclassical limit of the modified KP hierarchy, J. Phys. A: Math. Gen 23 (1990) 871-886.

[27] D.R. Lebedev and Yu.I. Manin, Conservation laws and Lax representation for Benney's long wave equations, Phys. lett. 74A (1979) 154-156.

[28] M. Manas, On the $r$ th dispersionless Toda hierarchy: factorization problem, additional symmetries and some solutions, J. Phys. A: Math. Gen. 37 (2004) 9195-9224.

[29] M. Manas, $S$-functions, reductions and hodograph solutions of the $r$ th dispersionless modified KP and Dym hierarchies, J. Phys. A: Math. Gen. 37 (2004) 11191-11221.

[30] M. Manas, L. Martinez Alonso and E. Medina, Reductions and hodograph solutions of the dispersionless KP hierarchy, J. Phys. A: Math. Gen. 35 (2002) 401-417.

[31] V.G. Mikhalev, Hamiltonian formalism of Korteweg-de Vries-type hierarchies. (Russian) Funktsional. Anal. i Prilozhen. 26, no. 2 (1992) 79-82; translation in Funct. Anal. Appl. 26, no. 2 (1992) 140-142.

[32] R.M. Miura, Conservation laws for fully nonlinear long wave equations, Stud. Appl. Math. 53 (1974) 45-56.

[33] M.V. Pavlov, Integrable hydrodynamic chains, J. Math. Phys. 44, no. 9 (2003) 4134-4156.

[34] M.V. Pavlov, Classification of integrable Egorov hydrodynamic chains, Teoret. Mat. Fiz. 138, no. 1 (2004) 55-70.

[35] M.V. Pavlov, S.I. Svinolupov and R.A. Sharipov, An invariant criterion for hydrodynamic integrability, Funktsional. Anal. i Prilozhen. 30 (1996) 18-29; translation in Funct. Anal. Appl. 30 (1996) 15-22.

[36] Z. Peradzyński, Nonlinear plane $k$-waves and Riemann invariants, Bull. Acad. Polon. Sci. Sr. Sci. Tech. 19 (1971) 625-632.

[37] B. Sévennec, Géométrie des systèmes hyperboliques de lois de conservation, Mémoire (nouvelle série) N56, Supplément au Bulletin de la Société Mathématique de France 122 (1994) $1-125$.

[38] L. Martinez Alonso and A.B. Shabat, Towards a theory of differential constraints of a hydrodynamic hierarchy, J. Nonlinear Math. Phys. 10, no. 2 (2003) 229-242.

[39] L. Martinez Alonso and A.B. Shabat, Energy-dependent potentials revisited: A universal hierarchy of hydrodynamic type, Phys. lett. A 299, no. 4 (2002) 359-365; Phys. Lett. A 300, no. 1 (2002) 58-64. 
[40] V.M. Teshukov, On the hyperbolicity of long wave equations, Dokl. Akad. Nauk SSSR 284, no. 3 (1985) 555-559.

[41] V.M. Teshukov, Characteristics, conservation laws and symmetries of the kinetic equations of motion of bubbles in a fluid, Prikl. Mekh. Tekhn. Fiz. 40, no. 2 (1999) 86-100; translation in J. Appl. Mech. Tech. Phys. 40, no. 2 (1999) 263-275.

[42] S.P. Tsarev, Geometry of Hamiltonian systems of hydrodynamic type. Generalized hodograph method, Izvestija AN USSR Math. 54, no. 5 (1990) 1048-1068.

[43] V.E. Zakharov, Benney equations and quasiclassical approximation in the inverse problem method, Funktsional. Anal. i Prilozhen. 14, no. 2 (1980) 15-24.

[44] V.E. Zakharov, On the Benney equations, Physica 3D, no. 1,2 (1981) 193-202. 